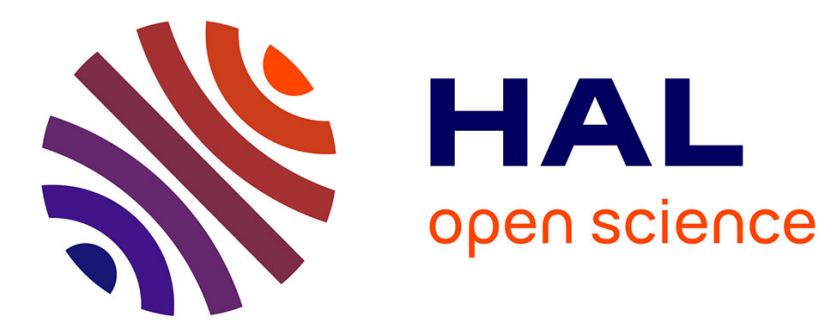

\title{
Post-processing of the planewave approximation of Schrödinger equations. Part I: linear operators
}

Eric Cancès, Geneviève Dusson, Yvon Maday, Benjamin Stamm, Martin Vohralík

\section{- To cite this version:}

Eric Cancès, Geneviève Dusson, Yvon Maday, Benjamin Stamm, Martin Vohralík. Post-processing of the planewave approximation of Schrödinger equations. Part I: linear operators. IMA Journal of Numerical Analysis, 2021, 41 (4), pp.2423-2455. 10.1093/imanum/draa044 . hal-01908039v4

\section{HAL Id: hal-01908039 \\ https://hal.science/hal-01908039v4}

Submitted on 17 Mar 2020

HAL is a multi-disciplinary open access archive for the deposit and dissemination of scientific research documents, whether they are published or not. The documents may come from teaching and research institutions in France or abroad, or from public or private research centers.
L'archive ouverte pluridisciplinaire HAL, est destinée au dépôt et à la diffusion de documents scientifiques de niveau recherche, publiés ou non, émanant des établissements d'enseignement et de recherche français ou étrangers, des laboratoires publics ou privés. 


\title{
Post-processing of the planewave approximation of Schrödinger equations. Part I: linear operators
}

\author{
ERIC CANCÈS
}

CERMICS, Ecole des Ponts, Université Paris-Est, 6 \& 8 Av. Pascal, 77455

Marne-la-Vallée, France and

Inria, 2 Rue Simone Iff, 75589 Paris, France

Geneviève Dusson*

Université Bourgogne Franche-Comté, Laboratoire de Mathématiques de Besançon, UMR CNRS 6623, Besançon, France

YVON MADAY

Sorbonne Université, Université Paris-Diderot SPC, CNRS, Laboratoire Jacques-Louis

Lions (LJLL), F-75005 Paris, France, and

Institut Universitaire de France, 75005 Paris, France

Benjamin Stamm

Center for Computational Engineering Science, RWTH Aachen University, Aachen, Germany

AND

MARTIN VOHRALÍK

Inria, 2 Rue Simone Iff, 75589 Paris, France and

CERMICS, Ecole des Ponts, Université Paris-Est, 6 \& 8 Av. Pascal, 77455

Marne-la-Vallée, France

\begin{abstract}
In this article, we prove a priori error estimates for the perturbation-based post-processing of the plane-wave approximation of Schrödinger equations introduced and tested numerically in previous works $[6,7]$. We consider here a Schrödinger operator $\mathcal{H}=-\frac{1}{2} \Delta+\mathcal{V}$ on $L^{2}(\Omega)$, where $\Omega$ is a cubic box with periodic boundary conditions, and where $\mathcal{V}$ is a multiplicative operator by a regular-enough function $\mathcal{V}$. The quantities of interest are, on the one hand, the ground-state energy defined as the sum of the lowest $N$ eigenvalues of $\mathcal{H}$, and, on the other hand, the ground-state density matrix, that is the spectral projector on the vector space spanned by the associated eigenvectors. Such a problem is central in first-principle molecular simulation, since it corresponds to the so-called linear subproblem in Kohn-Sham density functional theory (DFT). Interpreting the exact eigenpairs of $\mathcal{H}$ as perturbations of the numerical eigenpairs obtained by a variational approximation in a plane-wave (i.e. Fourier) basis, we compute first-order corrections for the eigenfunctions, which are turned into corrections on the ground-state density matrix. This allows us to increase the accuracy by a factor proportional to the inverse of the kinetic energy cutoff $E_{\mathrm{c}}{ }^{-1}$ of both the ground-state energy and the ground-state density matrix in Hilbert-Schmidt norm at
\end{abstract}

\footnotetext{
${ }^{*}$ Corresponding author. Email: genevieve.dusson@math.cnrs.fr
} 
a low computational extra-cost. Indeed, the computation of the corrections only requires the computation of the residual of the solution in a larger plane-wave basis and two Fast Fourier Transforms per eigenvalue. Schrödinger operator, perturbation method, planewave approximation, eigenvalue problem, post-processing.

\section{Introduction}

First-principle molecular simulation is a major tool to predict the properties of matter from the atomic to the macroscopic scales. It is widely used in different fields such as chemistry, condensed matter physics, or materials science. As a main advantage, it requires no empirical parameter except a few fundamental constants of physics (the reduced Planck constant $\hbar$, the electron mass $m_{e}$, the elementary charge $e$, the dielectric permittivity of the vacuum $\varepsilon_{0}$, the Boltzmann constant $k_{B}$ ), as well as the masses and atomic numbers of the nuclei contained in the system under consideration.

At this scale, matter is described as a system of $M$ nuclei and $N$ electrons. In the Born-Oppenheimer approximation [1], the nuclei, which are thousands times heavier than electrons, are considered as point-like classical particles, and the electrons are supposed to be, at each time $t$, in their ground-state. The resulting many-body Schrödinger equation describing the electronic ground state, a partial differential equation on a $3 \mathrm{~N}$-dimensional space, is way too costly to be solved in practice, hence approximations have to be resorted to, starting with considering a simpler model.

Many different approaches have been proposed to compute the electronic ground-state, among which wavefunction methods (see [12] and [4] for a mathematical introduction), density functional theory consisting of orbital-free and Kohn-Sham models $[9,16]$ and quantum Monte Carlo methods [17, 18].

The Kohn-Sham models are among the most widely used in physics and chemistry, as they provide a good compromise between accuracy and computational cost, being posed only in a 3-dimensional space. In condensed matter physics and materials science, most Kohn-Sham calculations are performed in a rectangular box $\Omega$, called a supercell, with periodic boundary conditions (Born-von Karman PBC). The most common method to discretize the Kohn-Sham equations then is to use a variational approximation in a plane-wave (Fourier) basis. For very large systems, unfortunately, using a fine discretization basis is too expensive, while using a coarse discretization basis leads to insufficiently accurate results.

In order to limit the computational cost of the method while preserving the quality of the numerical results, several post-processing methods have been proposed. Usually, the approach is to perform a full computation in a coarse basis, for which the computational cost is not excessively high, and then to make some not-too-expensive computation in a finer basis leading to a substantial improvement in accuracy.

In general, our approach falls into the category of two-grid methods, which have been applied to the case of a linear eigenvalue problem originally in [22], and nonlinear eigenvalue problems in [2, 13, 19]. In [22], the proposed method contains two steps: first, a linear eigenvalue problem is solved on a coarse finite element basis, and second, the computed eigenvectors are improved through a post-processing step, consisting of solving a boundary problem on a fine basis. The latter allows to increase the convergence rate of the eigenvectors in energy norm by at most the square of the discretization parameter of the coarse basis. For the nonlinear eigenvalue problems treated in $[2,13,19]$, the two steps are similar: first a nonlinear eigenvalue problem is solved on a coarse basis, then a boundary problem is solved on a fine basis. In [2], an alternative approach for the post-processing step consisting of solving a linear eigenvalue problem is also proposed. Also, a preprocessing step is added in [13] in order to better choose the coarse basis. In terms of improvement, the convergence rate of the post-processed eigenvectors in energy norm is doubled compared to the coarse quantities in [19] for the Hartree-Fock problem. It is improved by the square of the coarse discretization parameter in [2], and by its cube in [13], thanks to the proposed preprocessing step.

In $[6,7]$, we introduced a new post-processing method for periodic Kohn-Sham calculations in a planewave basis, leading to a significant gain in accuracy at a very limited computational extra-cost. This approach is based on the Rayleigh-Schrödinger perturbation method, considering the exact Kohn-Sham ground-state as a perturbation of the approximate ground-state computed in a finite basis set. Theoretical estimates in the asymptotic regime for the energy were announced and illustrated by numerical simulations. These 
simulations showed that the accuracy on the ground-state energy could be improved by a factor of 10 to 100 at a very limited extra-cost (about $3 \%$ ).

Compared to the existing post-processing methods $[2,13,19]$, we avoid here the resolution of a boundary value problem or an eigenvalue problem in the fine basis, because the considered operator to invert is diagonal. The gain in accuracy is proportional to the square of the plane-wave discretization parameter for the energy norm of the eigenvectors, which is comparable to what is obtained in [2] and [22]. Note that the proposed method is specific to a plane-wave discretisation. A theoretical and numerical comparison of these different approaches for nonlinear eigenvalue problems will be presented in the following article [11].

In this contribution, we focus on the linear subproblem of the Kohn-Sham model, and we present the proofs of the improved accuracy for the post-processing of the plane-wave approximation of Schrödinger equations stated in [7]. Corresponding proofs for the nonlinear Kohn-Sham model will be dealt with in a forthcoming paper [10].

The linear Kohn-Sham subproblem considered in this article consists in computing the rank $-N$ groundstate density matrix $\gamma_{0}$ of a linear Schrödinger operator $\mathcal{H}=-\frac{1}{2} \Delta+\mathcal{V}$, acting on the space $L_{\#}^{2}(\Omega)$ of realvalued square-integrable periodic functions on $\mathbb{R}^{3}$ with $\Omega$ as a periodic cell. For $\mathcal{V} \in L_{\#}^{2}(\Omega)$, the Hamiltonian $\mathcal{H}$ is diagonalizable in an orthonormal basis and its eigenvalues $\lambda_{1}^{0} \leq \lambda_{2}^{0} \leq \cdots$ (counting multiplicities) form a non-decreasing sequence of real numbers that tends to $+\infty$. Denoting by $\left(\phi_{i}^{0}\right)_{i>1}$ an orthonormal basis of associated eigenvectors, and assuming that there is a gap $g:=\lambda_{N+1}^{0}-\lambda_{N}^{0}>0$ between the $N^{\text {th }}$ and the $(N+1)^{\text {st }}$ eigenvalue of $\mathcal{H}$, the rank- $N$ ground-state density matrix is defined, using Dirac's bra-ket notation, as

$$
\gamma_{0}=\sum_{i=1}^{N}\left|\phi_{i}^{0}\right\rangle\left\langle\phi_{i}^{0}\right| .
$$

It is therefore the orthogonal projector on the $N$-dimensional vector subspace of $L_{\#}^{2}(\Omega)$ spanned by the eigenvectors associated with the lowest $N$ eigenvalues of $\mathcal{H}$. The ground-state energy is then defined as the scalar quantity

$$
\varepsilon_{0}=\sum_{i=1}^{N} \lambda_{i}^{0} .
$$

Note that $\mathcal{E}_{0}$ is not equal to the Kohn-Sham ground-state energy when $\mathcal{H}$ is the Kohn-Sham Hamiltonian due to nonlinear effects, called double-counting in the chemistry and physics literatures.

Our main result is summarized in Theorem 4.1. We show that, in the asymptotic regime where the discretization space is large enough, the convergence rates of both the post-processed ground-state density matrix (built from the post-processed eigenvectors defined in [6]) measured in Hilbert-Schmidt norm and the post-processed ground-state energy are improved by a factor proportional to $N_{\mathrm{c}}^{-2}$ where $N_{\mathrm{c}}$ is the discretization parameter. Since the kinetic energy cutoff $E_{\mathrm{c}}$ is itself proportional to $N_{\mathrm{c}}^{2}$, this improvement factor corresponds to $E_{\mathrm{c}}{ }^{-1}$, i.e. the inverse of the kinetic energy cutoff.

Note that we do not make any non-degeneracy assumption on the lowest $N$ eigenvalues of $\mathcal{H}$; only the presence of a positive gap between the $N^{\text {th }}$ and the $(N+1)^{\text {st }}$ eigenvalues of $\mathcal{H}$ is required. As in [6, 7], our approach strongly relies on the fact that, in plane-wave approximations, the kinetic energy operator $-\frac{1}{2} \Delta$, which is the leading term in the Hamiltonian $\mathcal{H}$, commutes with the orthogonal projector on the discretization space. The constant $C$ appearing in the estimations is generic and may depend on $N$ but is always independent of the discretization parameter $N_{\mathrm{c}}$.

This article is organized as follows. In Section 1, we summarize the main notation used in the article. In Section 2.1, we present in detail the linear subproblem of the Kohn-Sham model, as well as the characterization of $\gamma_{0}$ as the unique solution to some constrained optimization problem, and some other useful classical results. In Section 2.3, we describe the plane-wave discretization of this optimization problem. In Section 2.4, we translate the a priori error analysis results of [3] into the density matrix formalism. Our post-processing method based on Rayleigh-Schrödinger perturbation theory is described in Section 3. In Section 4.1, we present the main results of this paper, i.e. an improved convergence rate on the post-processed ground-state 
density matrix and energy. The proofs are given in Section 4.2. Some numerical simulations are presented in Section 5.

\section{List of notation}

To help the reader navigate through the paper, we summarize below the principal notation, and refer to the definitions when needed.

To start with, $N$ denotes the number of computed eigenvalues. The quantities related to the choice of discretisation (Section 2.3) are

- $E_{\mathrm{c}}$ : kinetic energy cutoff,

- $N_{\mathrm{c}}=\sqrt{\frac{E_{\mathrm{c}}}{2}} \frac{L}{\pi}:$ discretisation parameter,

- $X_{N_{\mathrm{c}}}$ : discretisation space.

The quantities related to energies are

- $\varepsilon_{0}$ : exact ground state energy (Section 2.1),

- $\mathcal{E}_{0, N_{\mathrm{c}}}$ : variational approximation of the ground state energy (2.19),

- $\widetilde{\mathcal{E}_{0, N_{\mathrm{c}}}}$ : perturbed ground state energy (3.10).

The different eigenfunctions, corresponding eigenvalues and Lagrange multiplier matrices defined in this article are

- $\Phi^{0}=\left(\phi_{1}^{0}, \cdots, \phi_{N}^{0}\right)^{T}$ and $\left(\lambda_{1}^{0}, \cdots, \lambda_{N}^{0}\right)$ : lowest $N$ eigenfunctions and corresponding eigenvalues of the Hamiltonian. The matrix of Lagrange multipliers $\Lambda^{0}$ is diagonal $\Lambda^{0}=\operatorname{diag}\left(\lambda_{1}^{0}, \cdots, \lambda_{N}^{0}\right)$.

- $\Phi_{N_{\mathrm{c}}}:=\left(\phi_{1, N_{\mathrm{c}}}, \ldots, \phi_{N, N_{\mathrm{c}}}\right)^{T}$ and $\left(\lambda_{1, N_{\mathrm{c}}}, \ldots, \lambda_{N, N_{\mathrm{c}}}\right)$ : lowest eigenfunctions diagonalizing the Hamiltonian on the discretisation space and corresponding eigenvalues (Section 2.3). The corresponding matrix of Lagrange multiplier is diagonal as well.

- $\Phi_{N_{\mathrm{c}}}^{0}=\left(\phi_{1, N_{\mathrm{c}}}^{0}, \ldots, \phi_{N, N_{\mathrm{c}}}^{0}\right)^{T}$ : eigenfunctions given by a unitary transform of $\Phi_{N_{\mathrm{c}}}$ such that $\Phi_{N_{\mathrm{c}}}^{0}$ is as much aligned with $\Phi^{0}$ as possible. The corresponding matrix of Lagrange multipliers $\Lambda_{N_{\mathrm{c}}}^{0}=$ $\left(\lambda_{i j, N_{\mathrm{c}}}^{0}\right)_{1 \leq i, j \leq N}:=\left(\left\langle\phi_{i, N_{\mathrm{c}}}^{0}|\mathcal{H}| \phi_{j, N_{\mathrm{c}}}^{0}\right\rangle\right)_{1 \leq i, j \leq N} \in \mathbb{R}^{N \times N}$ is not diagonal.

- $\left(\widetilde{\phi_{1, N_{\mathrm{c}}}}, \ldots, \widetilde{\phi_{N, N_{\mathrm{c}}}}\right)$ and $\left(\widetilde{\lambda_{1, N_{\mathrm{c}}}}, \ldots, \widetilde{\lambda_{N, N_{\mathrm{c}}}}\right)$ : perturbed eigenfunctions and perturbed eigenvalues (Definition 3.1).

The different density matrices involved in the following are:

- $\gamma_{0}$ : exact ground state density matrix (Section 2.1),

- $\gamma_{0, N_{\mathrm{c}}}$ : approximate density matrix (2.21),

- $\widetilde{\gamma_{0, N_{\mathrm{c}}}}$ : perturbed density matrix (Definition 3.1). Note that this density matrix is not an orthogonal projector, as mentioned in Remark 3.1. 


\section{Post-processing for the Kohn-Sham linear subproblem}

In order to simplify the notation, we consider a cubic lattice $\mathcal{R}=L \mathbb{Z}^{3}(L>0)$ corresponding to the supercell $\Omega=[0, L)^{3}$, but all our arguments straightforwardly apply to the general case of a lattice with lower or no point symmetry. For $1 \leq p \leq \infty$ and $s \in \mathbb{R}_{+}$, we denote by

$$
\begin{aligned}
L_{\#}^{p}(\Omega) & :=\left\{u \in L_{\mathrm{loc}}^{p}\left(\mathbb{R}^{3}, \mathbb{R}\right) \mid u \text { is } \mathcal{R} \text {-periodic }\right\}, \\
H_{\#}^{s}(\Omega) & :=\left\{u \in H_{\mathrm{loc}}^{s}\left(\mathbb{R}^{3}, \mathbb{R}\right) \mid u \text { is } \mathcal{R} \text {-periodic }\right\},
\end{aligned}
$$

the spaces of real-valued $\mathcal{R}$-periodic $L^{p}$ and $H^{s}$ functions, and by $\mathcal{L}\left(L_{\#}^{2}\right)$ the vector space of the bounded linear operators on $L_{\#}^{2}(\Omega)$.

\subsection{Problem setting}

Let $N \in \mathbb{N}^{*}$ and $\mathcal{V} \in L_{\#}^{2}(\Omega)$. In Kohn-Sham models, $N$ is the number of electrons (or of electron pairs in closed-shell models) in the simulation cell, and $\mathcal{V}$ is an approximation of the Kohn-Sham effective potential. Let $\mathcal{H}$ be the operator on $L_{\#}^{2}(\Omega)$ with domain $H_{\#}^{2}(\Omega)$ defined by

$$
\forall u \in H_{\#}^{2}(\Omega), \quad \mathcal{H} u=-\frac{1}{2} \Delta u+\mathcal{V} u .
$$

Since the operator $\mathcal{H}$ is a perturbation of the laplace operator on a torus, it is self-adjoint, bounded below, with compact resolvent [21]. It can therefore be diagonalized in an orthonormal basis: there exists a non-decreasing sequence $\left(\lambda_{i}^{0}\right)_{i \geq 1}$ of real numbers and an orthonormal basis $\left(\phi_{i}^{0}\right)_{i \geq 1}$ of $L_{\#}^{2}(\Omega)$ consisting of functions of $H_{\#}^{2}(\Omega)$ such that

$$
\forall i \geq 1, \quad \mathcal{H} \phi_{i}^{0}=\lambda_{i}^{0} \phi_{i}^{0} .
$$

We denote by $\Phi^{0}=\left(\phi_{1}^{0}, \cdots, \phi_{N}^{0}\right)^{T}$ and $\Lambda^{0}=\operatorname{diag}\left(\lambda_{1}^{0}, \cdots, \lambda_{N}^{0}\right)$.

A key assumption for our analysis is the following:

Assumption 2.1. There is a gap between the $N^{\text {th }}$ and the $(N+1)^{\text {st }}$ eigenvalues of $\mathcal{H}$, i.e.

$$
g:=\lambda_{N+1}^{0}-\lambda_{N}^{0}>0 .
$$

We denote by $\epsilon_{\mathrm{F}}:=\frac{\lambda_{N}^{0}+\lambda_{N+1}^{0}}{2}$ the Fermi level. Note that, in this setting, any real number in the range $\left(\lambda_{N}^{0}, \lambda_{N+1}^{0}\right)$ is an admissible Fermi level.

As already mentioned in the introduction, the purpose of the linear subproblem is to compute two quantities of interest:

1. the ground-state density matrix

$$
\gamma_{0}:=\mathbb{1}_{\left(-\infty, \epsilon_{\mathrm{F}}\right]}(\mathcal{H})=\sum_{i=1}^{N}\left|\phi_{i}^{0}\right\rangle\left\langle\phi_{i}^{0}\right| ;
$$

2. the ground-state energy

$$
\mathcal{E}_{0}:=\operatorname{Tr}\left(\mathcal{H} \gamma_{0}\right)=\sum_{i=1}^{N} \lambda_{i}^{0},
$$

where $\operatorname{Tr}$ denotes the trace, and will be properly introduced in Section 2.2.

The linear subproblem can be formulated as a variational problem in several ways. First, introducing the quadratic form

$$
H_{\#}^{1}(\Omega) \ni \psi \mapsto\langle\psi|\mathcal{H}| \psi\rangle:=\frac{1}{2} \int_{\Omega}|\nabla \psi|^{2}+\int_{\Omega} \nu|\psi|^{2} \in \mathbb{R}
$$


associated with $\mathcal{H}$, the energy functional $\mathcal{E}$ defined by

$$
\forall \Psi=\left(\psi_{1}, \ldots, \psi_{N}\right)^{T} \in\left[H_{\#}^{1}(\Omega)\right]^{N}, \quad \mathcal{E}(\Psi):=\sum_{i=1}^{N}\left\langle\psi_{i}|\mathcal{H}| \psi_{i}\right\rangle=\sum_{i=1}^{N}\left(\frac{1}{2} \int_{\Omega}\left|\nabla \psi_{i}\right|^{2}+\int_{\Omega} \mathcal{V}\left|\psi_{i}\right|^{2}\right),
$$

and the (infinite-dimensional) Stiefel manifold

$$
\mathcal{M}=\left\{\Psi=\left(\psi_{1}, \ldots, \psi_{N}\right)^{T} \in\left[H_{\#}^{1}(\Omega)\right]^{N} \mid \forall i, j=1, \ldots, N, \int_{\Omega} \psi_{i} \psi_{j}=\delta_{i j}\right\},
$$

we have

$$
\mathcal{E}_{0}=\inf \{\mathcal{E}(\Psi), \Psi \in \mathcal{M}\} .
$$

Besides, $\Phi^{0}=\left(\phi_{1}^{0}, \ldots, \phi_{N}^{0}\right)^{T}$ is a minimizer of (2.5). Note that $\Phi^{0}$ is not the unique minimizer of (2.5). Indeed, denoting by $\mathcal{O}(N):=\left\{U \in \mathbb{R}^{N \times N} \mid U^{T} U=1_{N}\right\}$ the orthogonal group in dimension $N$ ( $1_{N}$ is the identity matrix of rank $N$ ), we have

$$
\forall \Psi \in \mathcal{M}, \quad \forall U \in \mathcal{O}(N), \quad U \Psi \in \mathcal{M}, \quad \text { and } \quad \mathcal{E}(U \Psi)=\mathcal{E}(\Psi) .
$$

Therefore, $U \Phi^{0}$ is a minimizer of $(2.5)$ for all $U \in \mathcal{O}(N)$. In fact, under Assumption 2.1, the set of minimizers of (2.5) is exactly equal to $\mathcal{O}(N) \Phi^{0}$. For the sake of completeness, let us recall the proof of this elementary, but key, property. Let $\Psi=\left(\psi_{1}, \cdots, \psi_{N}\right)^{T}$ be a critical point of (2.5). The first-order optimality conditions satisfied by $\Psi$ read

$$
\forall i, j=1, \ldots, N, \quad \mathcal{H} \psi_{i}=\sum_{j=1}^{N} \lambda_{i j} \psi_{j}, \quad \int_{\Omega} \psi_{i} \psi_{i}=\delta_{i j}
$$

The $N \times N$ symmetric matrix $\Lambda=\left(\lambda_{i j}\right)_{i, j=1, \ldots, N}$ is the Lagrange multiplier of the matrix constraint $\int_{\Omega} \psi_{i} \psi_{j}=$ $\delta_{i j}$. It is not diagonal in general. On the other hand, since it is symmetric, there exists $U \in \mathcal{O}(N)$ such that $U \Lambda U^{T}=\operatorname{diag}\left(\lambda_{1}, \cdots, \lambda_{N}\right)$ with $\lambda_{1} \leq \lambda_{2} \leq \cdots \leq \lambda_{N}$. Then, $\Phi=\left(\phi_{1}, \cdots, \phi_{N}\right)^{T}:=U \Psi$ also is a critical point of (2.5) with the same energy as $\Psi$, and we have

$$
\forall 1 \leq i, j \leq N, \quad \mathcal{H} \phi_{i}=\lambda_{i} \phi_{i}, \quad \int_{\Omega} \phi_{i} \phi_{j}=\delta_{i j} \quad \text { and } \quad \mathcal{E}(\Phi)=\mathcal{E}(\Psi)=\sum_{i=1}^{N} \lambda_{i}
$$

For $\Psi$ to be a minimizer of (2.5), we must have $\lambda_{i}=\lambda_{i}^{0}$ for all $1 \leq i \leq N$. Under Assumption 2.1, we have in addition $\operatorname{Span}\left(\psi_{1}, \cdots, \psi_{N}\right)=\operatorname{Span}\left(\phi_{1}, \cdots, \phi_{N}\right)=\operatorname{Span}\left(\phi_{1}^{0}, \cdots, \phi_{N}^{0}\right)=\operatorname{Ran}\left(\gamma_{0}\right)$. Therefore, there exists $U \in \mathcal{O}(N)$ such that $\Psi=U \Phi_{0}$.

To get rid of the gauge invariance (2.6), it is possible to identify the elements of the Stiefel manifold (2.4) spanning the same subspace, introducing the Grassmann manifold as the quotient of the Stiefel manifold $\mathcal{M} / \sim$ with respect to the equivalence relation $\Psi \sim \tilde{\Psi}$ if $\tilde{\Psi}=U \Psi$ for some $U \in \mathcal{O}(N)$. Alternatively, as there exists a one-to-one correspondence between the subspace spanned by $\Psi \in \mathcal{M}$ and the orthogonal projector onto this space, it is possible to define the Grassmann manifold in terms of the orthogonal projectors called density matrices, and to reformulate problem (2.5) in terms of these density matrices. To this end, we define the (infinite-dimensional) Grassmann manifold

$$
\Upsilon=\left\{\gamma \in \mathcal{L}\left(L_{\#}^{2}\right) \mid \gamma^{*}=\gamma, \gamma^{2}=\gamma, \operatorname{Tr}(\gamma)=N, \operatorname{Tr}(-\Delta \gamma)<\infty\right\},
$$

its convex hull

$$
\mathcal{K}=\left\{\gamma \in \mathcal{L}\left(L_{\#}^{2}\right) \mid \gamma^{*}=\gamma, 0 \preceq \gamma \preceq 1, \operatorname{Tr}(\gamma)=N, \operatorname{Tr}(-\Delta \gamma)<\infty\right\},
$$

and the energy functional $E$ defined on $\mathcal{K}$ by

$$
\forall \gamma \in \mathcal{K}, \quad E(\gamma)=\operatorname{Tr}(\mathcal{H} \gamma)
$$


There holds

$$
\mathcal{E}_{0}=\inf \{E(\gamma), \gamma \in \Upsilon\}
$$

and

$$
\mathcal{E}_{0}=\inf \{E(\gamma), \gamma \in \mathcal{K}\} .
$$

Besides, under Assumption 2.1, $\gamma_{0}$ is the unique minimizer of both (2.10) and (2.11). Here $\gamma^{*}$ denotes the adjoint of $\gamma, 0 \leq \gamma \leq 1$ means $\forall u \in L_{\#}^{2}(\Omega), 0 \leq\langle u \mid \gamma u\rangle \leq\|u\|_{L_{\#}^{2}}^{2}$. The precise meaning of the terms $\operatorname{Tr}(-\Delta \gamma)$ and $\operatorname{Tr}(\mathcal{H} \gamma)$, as well as the proof of the fact that $\gamma_{0}$ is the unique minimizer of (2.10) and (2.11), will be given in the next section.

\section{$2.2 \quad$ Functional setting}

We denote by $\|\cdot\|$ the operator norm on $\mathcal{L}\left(L_{\#}^{2}\right)$, the space of bounded linear operators on $L_{\#}^{2}(\Omega)$. We also need to introduce the Banach space $\mathfrak{S}_{1}\left(L_{\#}^{2}\right)$ of trace-class operators on $L_{\#}^{2}(\Omega)$ and the Hilbert space $\mathfrak{S}_{2}\left(L_{\#}^{2}\right)$ of Hilbert-Schmidt operators on $L_{\#}^{2}(\Omega)$, respectively endowed with the norm defined by $\|A\|_{\mathfrak{S}_{1}\left(L_{\#}^{2}\right)}:=$ $\operatorname{Tr}(|A|)=\operatorname{Tr}\left(\sqrt{A^{*} A}\right)$ and the inner product defined by $(A, B)_{\mathfrak{S}_{2}\left(L_{\#}^{2}\right)}:=\operatorname{Tr}\left(A^{*} B\right)$. We refer to [20, Chapter VI] for an introduction to trace-class and Hilbert-Schmidt operators. Let us just recall here the properties which will be used in the sequel:

- for any orthonormal basis $\left(e_{n}\right)_{n \in \mathbb{N}}$ of $L_{\#}^{2}(\Omega)$, we have

$$
\begin{aligned}
& \forall A \in \mathfrak{S}_{1}\left(L_{\#}^{2}\right), \quad \operatorname{Tr}(A)=\sum_{n \in \mathbb{N}}\left\langle e_{n} \mid A e_{n}\right\rangle, \\
& \forall A \in \mathfrak{S}_{2}\left(L_{\#}^{2}\right), \quad\|A\|_{\mathfrak{S}_{2}\left(L_{\#}^{2}\right)}=\operatorname{Tr}\left(A^{*} A\right)^{1 / 2}=\left(\sum_{n \in \mathbb{N}}\left\|A e_{n}\right\|_{L_{\#}^{2}}^{2}\right)^{1 / 2} .
\end{aligned}
$$

If $A \in \mathcal{L}\left(L_{\#}^{2}\right)$ is a positive operator, that is if for all $u \in L_{\#}^{2}(\Omega)$, there holds that $\langle u \mid A u\rangle \geq 0$, then the value of the sum

$$
\operatorname{Tr}(A):=\sum_{n \in \mathbb{N}}\left\langle e_{n} \mid A e_{n}\right\rangle \in \mathbb{R}_{+} \cup\{+\infty\}
$$

is independent of the choice of the orthonormal basis $\left(e_{n}\right)_{n \in \mathbb{N}}$. If $A \in \mathcal{L}\left(L_{\#}^{2}\right)$ is positive and self-adjoint, then $A \in \mathfrak{S}_{1}\left(L_{\#}^{2}\right)$ if and only if $\operatorname{Tr}(A)<\infty$;

- $\mathfrak{S}_{1}\left(L_{\#}^{2}\right) \subset \mathfrak{S}_{2}\left(L_{\#}^{2}\right) \subset \mathcal{L}\left(L_{\#}^{2}\right)$ and for all $A \in \mathfrak{S}_{1}\left(L_{\#}^{2}\right)$,

$$
\|A\| \leq\|A\|_{\mathfrak{S}_{2}\left(L_{\#}^{2}\right)} \leq\|A\|_{\mathfrak{S}_{1}\left(L_{\#}^{2}\right)}
$$

- for all $A \in \mathfrak{S}_{1}\left(L_{\#}^{2}\right)$ and $B \in \mathcal{L}\left(L_{\#}^{2}\right)$, we have $A B \in \mathfrak{S}_{1}\left(L_{\#}^{2}\right), B A \in \mathfrak{S}_{1}\left(L_{\#}^{2}\right)$,

$$
\operatorname{Tr}(A B)=\operatorname{Tr}(B A), \quad\|A B\|_{\mathfrak{S}_{1}\left(L_{\#}^{2}\right)} \leq\|B\|\|A\|_{\mathfrak{S}_{1}\left(L_{\#}^{2}\right)}, \quad\|B A\|_{\mathfrak{S}_{1}\left(L_{\#}^{2}\right)} \leq\|B\|\|A\|_{\mathfrak{S}_{1}\left(L_{\#}^{2}\right)} ;
$$

- for all $A \in \mathfrak{S}_{1}\left(L_{\#}^{2}\right)$, there exists a unique function $\rho_{A} \in L_{\#}^{1}(\Omega)$, called the density associated with the operator $A$, such that for all $V \in L_{\#}^{\infty}(\Omega)$,

$$
\operatorname{Tr}(A V)=\int_{\Omega} \rho_{A} V
$$

where on the left-hand side of the above equality, $V \in \mathcal{L}\left(L_{\#}^{2}\right)$ denotes the multiplication operator by the function $V$; 
- for all $A \in \mathfrak{S}_{2}\left(L_{\#}^{2}\right)$ and $B \in \mathcal{L}\left(L_{\#}^{2}\right)$, we have $A B \in \mathfrak{S}_{2}\left(L_{\#}^{2}\right), B A \in \mathfrak{S}_{2}\left(L_{\#}^{2}\right)$,

$$
\|A B\|_{\mathfrak{S}_{2}\left(L_{\#}^{2}\right)} \leq\|B\|\|A\|_{\mathfrak{S}_{2}\left(L_{\#}^{2}\right)}, \quad\|B A\|_{\mathfrak{S}_{2}\left(L_{\#}^{2}\right)} \leq\|B\|\|A\|_{\mathfrak{S}_{2}\left(L_{\#}^{2}\right)}
$$

- for all $A \in \mathfrak{S}_{2}\left(L_{\#}^{2}\right)$ and $B \in \mathfrak{S}_{2}\left(L_{\#}^{2}\right), A B \in \mathfrak{S}_{1}\left(L_{\#}^{2}\right), B A \in \mathfrak{S}_{1}\left(L_{\#}^{2}\right)$,

$$
\operatorname{Tr}(A B)=\operatorname{Tr}(B A) \leq\|A\|_{\mathfrak{S}_{2}\left(L_{\#}^{2}\right)}\|B\|_{\mathfrak{S}_{2}\left(L_{\#}^{2}\right)}
$$

We set

$$
\begin{aligned}
& \forall \Psi=\left(\psi_{1}, \cdots, \psi_{N}\right)^{T} \in\left[L_{\#}^{2}(\Omega)\right]^{N}, \quad\|\Psi\|_{L_{\#}^{2}}:=\left(\sum_{i=1}^{N}\left\|\psi_{i}\right\|_{L_{\#}^{2}}^{2}\right)^{1 / 2}, \\
& \forall \Psi=\left(\psi_{1}, \cdots, \psi_{N}\right)^{T} \in\left[H_{\#}^{1}(\Omega)\right]^{N}, \quad\|\Psi\|_{H_{\#}^{1}}:=\left\|(1-\Delta)^{1 / 2} \Psi\right\|_{L_{\#}^{2}}=\left(\sum_{i=1}^{N}\left\|\psi_{i}\right\|_{H_{\#}^{1}}^{2}\right)^{1 / 2},
\end{aligned}
$$

and more generally, for any operator $A$ on $L_{\#}^{2}(\Omega)$ with domain $D(A)$,

$$
\forall \Psi \in[D(A)]^{N}, \quad\|A \Psi\|_{L_{\#}^{2}}:=\left(\sum_{i=1}^{N}\left\|A \psi_{i}\right\|_{L_{\#}^{2}}^{2}\right)^{1 / 2} .
$$

Let $\mathcal{R}^{*}=\frac{2 \pi}{L} \mathbb{Z}^{3}$ be the dual lattice of the periodic lattice $\mathcal{R}=L \mathbb{Z}^{3}$. For $\mathbf{k} \in \mathcal{R}^{*}$, we denote by $e_{\mathbf{k}}$ the plane-wave with wavevector $\mathbf{k}$, defined by

$$
\begin{aligned}
e_{\mathbf{k}}: \quad \mathbb{R}^{3} & \rightarrow \mathbb{C} \\
\mathbf{x} & \mapsto|\Omega|^{-1 / 2} e^{i \mathbf{k} \cdot \mathbf{x}},
\end{aligned}
$$

where $|\Omega|=L^{3}$. The family $\left(e_{\mathbf{k}}\right)_{\mathbf{k} \in \mathcal{R}^{*}}$ forms an orthonormal basis of the complex Hilbert space

$$
L_{\#}^{2}(\Omega, \mathbb{C}):=\left\{u \in L_{\mathrm{loc}}^{2}\left(\mathbb{R}^{3}, \mathbb{C}\right) \mid u \text { is } \mathcal{R} \text {-periodic }\right\},
$$

endowed with the scalar product

$$
\forall u, v \in L_{\#}^{2}(\Omega, \mathbb{C}), \quad\langle u \mid v\rangle=\int_{\Omega} \overline{u(\mathbf{r})} v(\mathbf{r}) d \mathbf{r},
$$

where $\overline{u(\mathbf{r})}$ denotes the complex conjugate of $u(\mathbf{r})$, and for all $v \in L_{\#}^{2}(\Omega, \mathbb{C})$,

$$
v(\mathbf{r})=\sum_{\mathbf{k} \in \mathcal{R}^{*}} \widehat{v}_{\mathbf{k}} e_{\mathbf{k}}(\mathbf{r}) \quad \text { with } \quad \widehat{v}_{\mathbf{k}}=\left\langle e_{\mathbf{k}} \mid v\right\rangle=|\Omega|^{-1 / 2} \int_{\Omega} v(\mathbf{r}) e^{-i \mathbf{k} \cdot \mathbf{r}} d \mathbf{r} .
$$

Recall that the periodic Sobolev spaces $H_{\#}^{s}(\Omega)$ can be characterized in a simple way using Fourier series: for $s \in \mathbb{R}$, we have

$$
H_{\#}^{s}(\Omega):=\left\{v=\left.\sum_{\mathbf{k} \in \mathcal{R}^{*}} \widehat{v}_{\mathbf{k}} e_{\mathbf{k}}\left|\forall \mathbf{k}, \quad \widehat{v}_{-\mathbf{k}}=\overline{\widehat{v}}_{\mathbf{k}}, \quad\|v\|_{H_{\#}^{s}}^{2}:=\sum_{\mathbf{k} \in \mathcal{R}^{*}}\left(1+|\mathbf{k}|^{2}\right)^{s}\right| \widehat{v}_{\mathbf{k}}\right|^{2}<\infty\right\},
$$

where the $H_{\#}^{s}$ inner product is defined by

$$
\forall u, v \in H_{\#}^{s}(\Omega), \quad(u, v)_{H_{\#}^{s}}:=\sum_{\mathbf{k} \in \mathcal{R}^{*}}\left(1+|\mathbf{k}|^{2}\right)^{s} \overline{u_{\mathbf{k}}} v_{\mathbf{k}} .
$$


Let us now clarify the meaning of the terms $\operatorname{Tr}(-\Delta \gamma)$ and $\operatorname{Tr}(\mathcal{H} \gamma)$ appearing in $(2.7)-(2.9)$. Let $\gamma \in \mathcal{L}\left(L_{\#}^{2}\right)$ be self-adjoint and positive. Since $|\nabla|$ (i.e. the multiplication operator by $|\mathbf{k}|$ in Fourier representation) is a bounded linear operator from $H_{\#}^{s}(\Omega)$ to $H_{\#}^{s-1}(\Omega),|\nabla| \gamma|\nabla|$ defines a bounded linear operator from $H_{\#}^{1}(\Omega)$ to $H_{\#}^{-1}(\Omega)$. If in addition, $\operatorname{Ran}(|\nabla| \gamma|\nabla|) \subset L_{\#}^{2}(\Omega)$ and

$$
\exists C \in \mathbb{R}_{+} \quad \text { such that } \quad \forall u \in H_{\#}^{1}(\Omega), \quad\||\nabla| \gamma|\nabla| u\|_{L_{\#}^{2}} \leq C\|u\|_{L_{\#}^{2}},
$$

then $|\nabla| \gamma|\nabla|$ can be uniquely extended to a bounded, self-adjoint, positive operator on $L_{\#}^{2}(\Omega)$, also denoted by $|\nabla| \gamma|\nabla|$ for simplicity. In this case, $\operatorname{Tr}(|\nabla| \gamma|\nabla|)$ is well-defined in $\mathbb{R}_{+} \cup\{+\infty\}$. In view of the fact that $-\Delta=|\nabla|^{2}$, the notation

$$
\operatorname{Tr}(-\Delta \gamma):=\operatorname{Tr}(|\nabla| \gamma|\nabla|)
$$

is commonly used in the mathematical physics literature. Let us emphasize that $\operatorname{Tr}(-\Delta \gamma)<\infty$ only means that $\operatorname{Tr}(|\nabla| \gamma|\nabla|)<\infty$; in particular, it does not imply that the operator $-\Delta \gamma$ is in $\mathfrak{S}_{1}\left(L_{\#}^{2}\right)$.

It follows from the Hoffmann-Ostenhof inequality [14] that for all $\gamma \in \mathcal{K}, \rho_{\gamma} \geq 0$ and $\sqrt{\rho_{\gamma}} \in H_{\#}^{1}(\Omega) \hookrightarrow$ $L_{\#}^{6}(\Omega)$. The real number $\operatorname{Tr}(\mathcal{H} \gamma)$ can therefore be defined for all $\gamma \in \mathcal{K}$ as

$$
\operatorname{Tr}(\mathcal{H} \gamma):=\frac{1}{2} \operatorname{Tr}(-\Delta \gamma)+\int_{\Omega} \rho_{\gamma} V
$$

It is known in addition (see e.g. [5]) that, under Assumption 2.1, there exist $0<c \leq C<\infty$ such that

$$
c(1-\Delta) \leq\left|\mathcal{H}-\epsilon_{\mathrm{F}}\right| \leq C(1-\Delta),
$$

where the operator $\left|\mathcal{H}-\epsilon_{\mathrm{F}}\right|$ is defined as $\left|\mathcal{H}-\epsilon_{\mathrm{F}}\right|=-\gamma_{0}\left(\mathcal{H}-\epsilon_{\mathrm{F}}\right) \gamma_{0}+\left(1-\gamma_{0}\right)\left(\mathcal{H}-\epsilon_{\mathrm{F}}\right)\left(1-\gamma_{0}\right)$, and can be written using the eigendecomposition of $\mathcal{H}$ as

$$
\left|\mathcal{H}-\epsilon_{\mathrm{F}}\right|=\sum_{i=1}^{+\infty}\left|\lambda_{i}^{0}-\epsilon_{\mathrm{F}}\right|\left|\phi_{i}^{0}\right\rangle\left\langle\phi_{i}^{0}\right|
$$

where $\left(\phi_{i}^{0}, \lambda_{i}^{0}\right)_{i \geq 1}$ are eigenpairs of $\mathcal{H}$ defined in (2.2). There also holds

$$
\forall \gamma \in \mathcal{K}, \quad \operatorname{Tr}(\mathcal{H} \gamma)-\operatorname{Tr}\left(\mathcal{H} \gamma_{0}\right)=\left\|\left|\mathcal{H}-\epsilon_{\mathrm{F}}\right|^{1 / 2}\left(\gamma-\gamma_{0}\right)\right\|_{\mathfrak{S}_{2}\left(L_{\#}^{2}\right)} .
$$

We deduce from (2.16) and (2.17) that there exist two constants $0<c \leq C<\infty$ such that

$$
\forall \gamma \in \mathcal{K}, \quad c\left\|(1-\Delta)^{1 / 2}\left(\gamma-\gamma_{0}\right)\right\|_{\mathfrak{S}_{2}\left(L_{\#}^{2}\right)}^{2} \leq E(\gamma)-\mathcal{E}_{0} \leq C\left\|(1-\Delta)^{1 / 2}\left(\gamma-\gamma_{0}\right)\right\|_{\mathfrak{S}_{2}\left(L_{\#}^{2}\right)}^{2} .
$$

This implies in particular that $\gamma_{0}$ is the unique minimizer of (2.10) and (2.11).

Note that for all $\gamma \in \Upsilon$, as $\gamma^{2}=\gamma$ and by the cyclicity of the trace, there also holds $\operatorname{Tr}(\mathcal{H} \gamma)=\operatorname{Tr}(\gamma \mathcal{H})=$ $\operatorname{Tr}(\gamma \mathcal{H} \gamma)$

\subsection{Discretization}

In order to solve problem (2.5) numerically, we use a plane-wave discretization. For each $\mathbf{k} \in \mathcal{R}^{*}$, the kinetic energy of the plane-wave $e_{\mathbf{k}}$ is given by $\frac{1}{2}|\mathbf{k}|^{2}$, where $|\cdot|$ denotes the Euclidean norm. To construct a discretization space, we introduce some energy cut-off $E_{\mathrm{c}}>0$ and consider all plane-waves whose kinetic energy is smaller than $E_{\mathrm{c}}$, i.e. $|\mathbf{k}| \leq \sqrt{2 E_{\mathrm{c}}}$. For each cut-off energy $E_{\mathrm{c}}$, we set $N_{\mathrm{c}}=\sqrt{\frac{E_{\mathrm{c}}}{2}} \frac{L}{\pi}$ and

$$
X_{N_{\mathrm{c}}}:=\left\{\sum_{\mathbf{k} \in \mathcal{R}^{*},|\mathbf{k}| \leq \frac{2 \pi}{L} N_{\mathrm{c}}} \widehat{v}_{\mathbf{k}} e_{\mathbf{k}} \mid \widehat{v}_{-\mathbf{k}}=\widehat{\widehat{v}}_{\mathbf{k}}, \forall \mathbf{k}\right\} \subset \bigcap_{s \in \mathbb{R}} H_{\#}^{s}(\Omega) .
$$


For all $s \in \mathbb{R}$, for all $r \leq s$, and for each $v \in H_{\#}^{s}(\Omega)$, the best approximation of $v$ in $X_{N_{\mathrm{c}}}$ in any $H_{\#}^{r}$-norm is

$$
\Pi_{N_{\mathrm{c}}} v=\sum_{\mathbf{k} \in \mathcal{R}^{*},|\mathbf{k}| \leq \frac{2 \pi}{L} N_{\mathrm{c}}} \widehat{v}_{\mathbf{k}} e_{\mathbf{k}}
$$

We denote by $\Pi_{N_{\mathrm{c}}}^{\perp}=\left(1-\Pi_{N_{\mathrm{c}}}\right)$ the orthogonal projector on $X_{N_{\mathrm{c}}}^{\perp}$, the orthogonal of $X_{N_{\mathrm{c}}}$. The variational approximation to the ground-state energy in $X_{N_{\mathrm{c}}}$ is defined as

$$
\mathcal{E}_{0, N_{\mathrm{c}}}=\inf \left\{\mathcal{E}\left(\Psi_{N_{\mathrm{c}}}\right), \Psi_{N_{\mathrm{c}}} \in \mathcal{M} \cap\left[X_{N_{\mathrm{c}}}\right]^{N}\right\},
$$

with $\mathcal{E}$ and $\mathcal{M}$ defined in (2.3) and (2.4). Let $\lambda_{1, N_{\mathrm{c}}} \leq \lambda_{2, N_{\mathrm{c}}} \leq \cdots \leq \lambda_{\operatorname{dim}\left(X_{N_{\mathrm{c}}}\right), N_{\mathrm{c}}}$ be the $\operatorname{dim}\left(X_{N_{\mathrm{c}}}\right)$ eigenvalues (counting multiplicites) of the Hermitian linear operator $\mathcal{H}_{N_{\mathrm{c}} \text {,proj }}: X_{N_{\mathrm{c}}} \rightarrow X_{N_{\mathrm{c}}}$ defined as

$$
\mathcal{H}_{N_{\mathrm{c}}, \operatorname{proj}}=\Pi_{N_{\mathrm{c}}} \mathcal{H} \Pi_{N_{\mathrm{c}}}=-\frac{1}{2} \Pi_{N_{\mathrm{c}}} \Delta \Pi_{N_{\mathrm{c}}}+\Pi_{N_{\mathrm{c}}} v \Pi_{N_{\mathrm{c}}} .
$$

Let $\left(\phi_{1, N_{\mathrm{c}}}, \ldots, \phi_{N, N_{\mathrm{c}}}\right)$ be an orthonormal family of eigenvectors of $\mathcal{H}_{N_{\mathrm{c}}, \text { proj }}$ associated with the eigenvalues $\lambda_{1, N_{\mathrm{c}}} \leq \cdots \leq \lambda_{N, N_{\mathrm{c}}}$ :

$$
\mathcal{H}_{N_{\mathrm{c}}, \operatorname{proj}} \phi_{i, N_{\mathrm{c}}}=\lambda_{i, N_{\mathrm{c}}} \phi_{i, N_{\mathrm{c}}}, \quad \int_{\Omega} \phi_{i, N_{\mathrm{c}}} \phi_{j, N_{\mathrm{c}}}=\delta_{i j}, \quad \forall 1 \leq i, j \leq N,
$$

and let $\Phi_{N_{\mathrm{c}}}:=\left(\phi_{1, N_{\mathrm{c}}}, \ldots, \phi_{N, N_{\mathrm{c}}}\right)^{T}$. Then $\Phi_{N_{\mathrm{c}}}$ is a minimizer of (2.19). Denoting by

$$
\gamma_{0, N_{\mathrm{c}}}=\sum_{i=1}^{N}\left|\phi_{i, N_{\mathrm{c}}}\right\rangle\left\langle\phi_{i, N_{\mathrm{c}}}\right|
$$

the associated density matrix, we have

$$
\varepsilon_{0, N_{\mathrm{c}}}=\operatorname{Tr}\left(\mathcal{H} \gamma_{0, N_{\mathrm{c}}}\right)=\sum_{j=1}^{N} \lambda_{j, N_{\mathrm{c}}} .
$$

\subsection{A priori results on the density matrices}

From now on, we make the following technical assumption:

Assumption 2.2. $\mathcal{V}$ is a $\mathcal{R}$-periodic potential satisfying $\mathcal{V} \in H_{\#}^{s}(\Omega)$ for $s>3 / 2$.

Note that this assumption implies that $\mathcal{V} \in L_{\#}^{\infty}(\Omega)$ and $\nabla \mathcal{V} \in L_{\#}^{3}(\Omega)$.

The a priori error estimates established in [3] for the nonlinear Kohn-Sham model also hold true for the linear subproblem. In order to use these results in the present setting, it is convenient to reformulate them in terms of density matrices. As in [3], we introduce

$$
\mathcal{M}^{\Phi^{0}}:=\left\{\Psi \in \mathcal{M} \mid\left\|\Psi-\Phi^{0}\right\|_{L_{\#}^{2}}=\min _{U \in \mathcal{O}(N)}\left\|U \Psi-\Phi^{0}\right\|_{L_{\#}^{2}}\right\}
$$

where $\Phi^{0}=\left(\phi_{1}^{0}, \ldots, \phi_{N}^{0}\right)^{T},\left(\phi_{1}^{0}, \ldots, \phi_{N}^{0}\right)$ being a family of orthonormal eigenvectors of $\mathcal{H}$ associated with the eigenvalues $\lambda_{1}^{0} \leq \cdots \leq \lambda_{N}^{0}$ fixed once and for all.

Proceeding as in [3], it can be shown that, for $N_{c}$ large enough, (2.19) has a unique minimizer $\Phi_{N_{\mathrm{c}}}^{0}=$ $\left(\phi_{1, N_{\mathrm{c}}}^{0}, \ldots, \phi_{N, N_{\mathrm{c}}}^{0}\right)^{T}$ belonging to $\mathcal{M}^{\Phi^{0}}$, that the set of minimizers of $(2.19)$ is $\mathcal{O}(N) \Phi_{N_{\mathrm{c}}}^{0}$, and that, consequently, all the minimizers of (2.19) share the same density matrix. In particular

$$
\gamma_{0, N_{\mathrm{c}}}=\sum_{i=1}^{N}\left|\phi_{i, N_{\mathrm{c}}}^{0}\right\rangle\left\langle\phi_{i, N_{\mathrm{c}}}^{0}\right| .
$$


We denote by

$$
\Lambda_{N_{\mathrm{c}}}^{0}=\left(\lambda_{i j, N_{\mathrm{c}}}^{0}\right)_{1 \leq i, j \leq N}:=\left(\left\langle\phi_{i, N_{\mathrm{c}}}^{0}|\mathcal{H}| \phi_{j, N_{\mathrm{c}}}^{0}\right\rangle\right)_{1 \leq i, j \leq N} \in \mathbb{R}^{N \times N}
$$

the Lagrange multiplier matrix of the orthonormality constraints. Note that the matrix $\Lambda_{N_{\mathrm{c}}}^{0}$ is not diagonal in general, but that we have

$$
\mathcal{E}_{0, N_{\mathrm{c}}}=\operatorname{Tr}\left(\Lambda_{N_{\mathrm{c}}}^{0}\right) .
$$

The following lemma allows one to translate the a priori results of [3, Theorem 4.2] in terms of density matrices.

Lemma 2.1. Under Assumption 2.1, there exist $0<c \leq C<\infty$ and $N_{\mathrm{c}}^{0} \in \mathbb{N}$, such that for all $N_{\mathrm{c}} \geq N_{\mathrm{c}}^{0}$,

$$
\begin{gathered}
\left\|\Phi_{N_{\mathrm{c}}}^{0}-\Phi^{0}\right\|_{L_{\#}^{2}} \leq\left\|\gamma_{0, N_{\mathrm{c}}}-\gamma_{0}\right\|_{\mathfrak{S}_{2}\left(L_{\#}^{2}\right)} \leq \sqrt{2}\left\|\Phi_{N_{\mathrm{c}}}^{0}-\Phi^{0}\right\|_{L_{\#}^{2}}, \\
c\left\|(1-\Delta)^{1 / 2}\left(\Phi_{N_{\mathrm{c}}}^{0}-\Phi^{0}\right)\right\|_{L_{\#}^{2}} \leq\left\|(1-\Delta)^{1 / 2}\left(\gamma_{0, N_{\mathrm{c}}}-\gamma_{0}\right)\right\|_{\mathfrak{S}_{2}\left(L_{\#}^{2}\right)} \leq C\left\|(1-\Delta)^{1 / 2}\left(\Phi_{N_{\mathrm{c}}}^{0}-\Phi^{0}\right)\right\|_{L_{\#}^{2}} .
\end{gathered}
$$

The proof is given in the Appendix.

We then immediately infer from [3, Theorem 4.2] that under Assumptions 2.1 and 2.2, there exist $C \in \mathbb{R}_{+}$ and $N_{\mathrm{c}}^{0} \in \mathbb{N}$ such that for all $N_{\mathrm{c}} \geq N_{\mathrm{c}}^{0}$,

$$
\begin{gathered}
\left\|\gamma_{0}-\gamma_{0, N_{\mathrm{c}}}\right\|_{\mathfrak{S}_{2}\left(L_{\#}^{2}\right)} \leq C N_{\mathrm{c}}^{-2}, \\
\left\|\gamma_{0}-\gamma_{0, N_{\mathrm{c}}}\right\|_{\mathfrak{S}_{2}\left(L_{\#}^{2}\right)} \leq C N_{\mathrm{c}}^{-1}\left\|(1-\Delta)^{1 / 2}\left(\gamma_{0}-\gamma_{0, N_{\mathrm{c}}}\right)\right\|_{\mathfrak{S}_{2}\left(L_{\#}^{2}\right)} .
\end{gathered}
$$

Moreover, there exists $C \in \mathbb{R}_{+}$such that

$$
\left\|\Lambda^{0}-\Lambda_{N_{\mathrm{c}}}^{0}\right\|_{\mathrm{F}} \leq C\left\|(1-\Delta)^{1 / 2}\left(\gamma_{0}-\gamma_{0, N_{\mathrm{c}}}\right)\right\|_{\mathfrak{S}_{2}\left(L_{\#}^{2}\right)}^{2},
$$

where $\|\cdot\|_{\mathrm{F}}$ denotes the Frobenius norm.

\section{Post-processing of the plane-wave approximation}

\subsection{A key remark}

Let us introduce the Hamiltonian on $L_{\#}^{2}(\Omega)$ with domain $H_{\#}^{2}(\Omega)$ defined by

$$
\forall u \in H_{\#}^{2}(\Omega), \quad \mathcal{H}_{N_{\mathrm{c}}} u=-\frac{1}{2} \Delta u+\Pi_{N_{\mathrm{c}}} v \Pi_{N_{\mathrm{c}}} u .
$$

Since $X_{N_{\mathrm{c}}}$ and $X_{N_{\mathrm{c}}}^{\perp}$ are invariant subspaces of $\mathcal{H}_{N_{\mathrm{c}}}$, the Hamiltonian $\mathcal{H}_{N_{\mathrm{c}}}$ can be represented in term of $\mathcal{H}_{N_{\mathrm{c}}, \text { proj }}$ as follows:

$$
\mathcal{H}_{N_{\mathrm{c}}}=\left(\begin{array}{c|c}
\hline \mathcal{H}_{N_{\mathrm{c}}, \operatorname{proj}} & 0 \\
0 & -\frac{1}{2} \Delta
\end{array}\right\} X_{X_{N_{\mathrm{c}}}} \underbrace{\perp}_{X_{N_{\mathrm{c}}}^{\perp}}\} X_{N_{\mathrm{c}}}^{\perp}
$$

The eigenvalues of the Laplace operator, which is diagonal in plane-wave bases, are explicitly known and its smallest eigenvalue on the invariant subspace $X_{N_{\mathrm{c}}}^{\perp}$ is larger than $\frac{1}{2}\left(\frac{2 \pi N_{\mathrm{c}}}{L}\right)^{2}$. Therefore, as soon as

$$
\lambda_{N, N_{\mathrm{c}}}<\frac{1}{2}\left(\frac{2 \pi N_{\mathrm{c}}}{L}\right)^{2},
$$


where we recall that $\lambda_{1, N_{\mathrm{c}}} \leq \cdots \leq \lambda_{N, N_{\mathrm{c}}}$ are the lowest $N$ eigenvalues (counting multiplicities) of the operator $\mathcal{H}_{N_{c} \text {,proj }}$ defined in $(2.20)$, we have

$$
\forall j=1, \ldots, N, \quad \mathcal{H}_{N_{\mathrm{c}}} \phi_{j, N_{\mathrm{c}}}=\lambda_{j, N_{\mathrm{c}}} \phi_{j, N_{\mathrm{c}}}, \quad \text { and } \quad \forall i, j=1, \ldots, N, \quad \int_{\Omega} \phi_{i, N_{\mathrm{c}}} \phi_{j, N_{\mathrm{c}}}=\delta_{i j},
$$

and $\lambda_{1, N_{\mathrm{c}}} \leq \cdots \leq \lambda_{N, N_{\mathrm{c}}}$ are also the lowest $N$ eigenvalues (counting multiplicities) of the operator $\mathcal{H}_{N_{\mathrm{c}}}$. A key observation is that the lowest energy eigenmodes of $\mathcal{H}$ satisfy

$$
\forall j=1, \ldots, N, \quad\left(\mathcal{H}_{N_{\mathrm{c}}}+\mathcal{V}_{N_{\mathrm{c}}}^{\perp}\right) \phi_{j}^{0}=\lambda_{j}^{0} \phi_{j}^{0}, \quad \text { and } \quad \forall i, j=1, \ldots, N, \quad \int_{\Omega} \phi_{i}^{0} \phi_{j}^{0}=\delta_{i j},
$$

where

$$
\mathcal{V}_{N_{\mathrm{c}}}^{\perp}=v-\Pi_{N_{\mathrm{c}}} v \Pi_{N_{\mathrm{c}}} .
$$

We can therefore apply the Rayleigh-Schrödinger perturbation method [15] using $\left(\phi_{j, N_{\mathrm{c}}}, \lambda_{j, N_{\mathrm{c}}}\right)_{j=1, \ldots, N}$ as the reference solution and $\left(\phi_{j}^{0}, \lambda_{j}^{0}\right)_{j=1, \ldots, N}$ as the perturbed solution, in order to build improved approximations of the orbitals and eigenvalues respectively denoted by $\left(\widetilde{\phi_{j, N_{\mathrm{c}}}}\right)_{j=1, \ldots, N}$ and $\left(\widetilde{\lambda_{j, N_{\mathrm{c}}}}\right)_{j=1, \ldots, N}$, as well as an improved density matrix $\widetilde{\gamma_{0, N_{\mathrm{c}}}}$ and improved energy $\widetilde{\varepsilon_{0, N_{\mathrm{c}}}}$.

\subsection{Corrections computation}

More precisely, we apply first-order perturbation to the analytic family of operators $\mathcal{H}(\beta)=\mathcal{H}_{N_{\mathrm{c}}}+\beta \mathcal{V}_{N_{\mathrm{c}}}^{\perp}$, where $\beta \in \mathbb{R}$ is a parameter, which amounts to considering $\mathcal{H}(0)=\mathcal{H}_{N_{\mathrm{c}}}$ and (3.3) as the unperturbed eigenvalue problem, and $\mathcal{H}(1)=\mathcal{H}$ and (3.4) as the perturbed eigenvalue problem. Assuming that the eigenvalues are not degenerate, we obtain at first order for the eigenfunctions, and at second order for the eigenvalues,

$$
\forall j=1, \ldots, N, \quad \phi_{j}^{0} \simeq \phi_{j, N_{\mathrm{c}}}^{0}+\phi_{j, N_{\mathrm{c}}}^{(1)}, \quad \lambda_{j}^{0} \simeq \lambda_{j, N_{\mathrm{c}}}+\lambda_{j, N_{\mathrm{c}}}^{(2)},
$$

where

$$
\phi_{j, N_{\mathrm{c}}}^{(1)}=-\left(-\frac{1}{2} \Delta-\lambda_{j, N_{\mathrm{c}}}\right)^{-1} r_{j} \in X_{N_{\mathrm{c}}}^{\perp}
$$

with $r_{j}$ being the residual

$$
r_{j}=\left(-\frac{1}{2} \Delta+\nu-\lambda_{j, N_{\mathrm{c}}}\right) \phi_{j, N_{\mathrm{c}}}=\left(\mathcal{H}_{N_{\mathrm{c}}}+\mathcal{V}_{N_{\mathrm{c}}}^{\perp}-\lambda_{j, N_{\mathrm{c}}}\right) \phi_{j, N_{\mathrm{c}}}=\mathcal{V}_{N_{\mathrm{c}}}^{\perp} \phi_{j, N_{\mathrm{c}}}
$$

and

$$
\lambda_{j, N_{\mathrm{c}}}^{(2)}=\left\langle\phi_{j, N_{\mathrm{c}}}^{(1)} \mid r_{j}\right\rangle=-\left\langle r_{j}\left|\left(-\frac{1}{2} \Delta-\lambda_{j, N_{\mathrm{c}}}\right)^{-1}\right| r_{j}\right\rangle
$$

We observe that the corrections on the eigenfunctions given in (3.6) are well-defined even if $\lambda_{j, N_{c}}$ is degenerate. We therefore define the perturbed eigenvectors, density matrix, and energy for the general case as follows.

Definition 3.1 (Perturbed eigenvectors, eigenvalues, density matrix, and energy). For all $N_{c} \geq N_{c}^{0}$ and all $j=1, \ldots, N$, the perturbed eigenvectors are defined as

$$
\widetilde{\phi_{j, N_{\mathrm{c}}}}=\phi_{j, N_{\mathrm{c}}}+\phi_{j, N_{\mathrm{c}}}^{(1)}
$$

the perturbed eigenvalues as

$$
\widetilde{\lambda_{j, N_{\mathrm{c}}}}=\lambda_{j, N_{\mathrm{c}}}+\lambda_{j, N_{\mathrm{c}}}^{(2)}
$$

the perturbed density matrix as

$$
\widetilde{\gamma_{0, N_{\mathrm{c}}}}=\gamma_{0, N_{\mathrm{c}}}+\gamma_{N_{\mathrm{c}}}^{(1)},
$$


with

$$
\gamma_{N_{\mathrm{c}}}^{(1)}=\sum_{j=1}^{N}\left|\phi_{j, N_{\mathrm{c}}}^{(1)}\right\rangle\left\langle\phi_{j, N_{\mathrm{c}}}\left|+\sum_{j=1}^{N}\right| \phi_{j, N_{\mathrm{c}}}\right\rangle\left\langle\phi_{j, N_{\mathrm{c}}}^{(1)}\right|,
$$

and the perturbed energy as

$$
\widetilde{\varepsilon_{0, N_{\mathrm{c}}}}=\sum_{j=1}^{N} \widetilde{\lambda_{j, N_{\mathrm{c}}}}=\operatorname{Tr}\left(\gamma_{0, N_{\mathrm{c}}} \mathcal{H} \widetilde{\gamma_{0, N_{\mathrm{c}}}}\right) .
$$

Remark 3.1. Note that even if we call $\widetilde{\gamma_{0, N_{\mathrm{c}}}}$ a density matrix, $\widetilde{\gamma_{0, N_{\mathrm{c}}}} \notin \mathcal{K}$ in general. Indeed, $\widetilde{\gamma_{0, N_{\mathrm{c}}}}=\widetilde{\gamma_{0, N_{\mathrm{c}}}} *$ and $\operatorname{Tr}\left(\widetilde{\gamma_{0, N_{\mathrm{c}}}}\right)=N$, but we do not have in general $0 \leq \widetilde{\gamma_{0, N_{\mathrm{c}}}} \leq 1$. Hence, the perturbed energy, which is defined as the sum of the perturbed eigenvalues, is not equal to the energy of the perturbed density matrix, i.e. $\widetilde{\mathcal{E}_{0, N_{\mathrm{c}}}} \neq \operatorname{Tr}\left(\mathcal{H} \widetilde{\gamma_{0, N_{\mathrm{c}}}}\right)$. Nevertheless definition (3.10) corresponds to the correction on the eigenvalues $\left(\lambda_{j, N_{\mathrm{c}}}\right)_{j=1, \ldots, N}$ given by perturbation theory at second-order as in [6], the contribution at first-order on the eigenvalues being zero.

Remark 3.2. As we shall see in Section 5, the quantities $\phi_{j, N_{\mathrm{c}}}^{(1)}$ are easily computable.

\section{Convergence improvement on the density matrix and the energy}

\subsection{Main results}

The main results of this article are collected in the following theorem.

Theorem 4.1. Under Assumptions 2.1-2.2, there exist $C \in \mathbb{R}_{+}$and $N_{\mathrm{c}}^{0} \in \mathbb{N}$, such that for all $N_{\mathrm{c}} \geq N_{\mathrm{c}}^{0}$,

$$
\left\|(1-\Delta)^{1 / 2}\left(\widetilde{\gamma_{0, N_{\mathrm{c}}}}-\gamma_{0}\right)\right\|_{\mathfrak{S}_{2}\left(L_{\#}^{2}\right)} \leq C N_{\mathrm{c}}^{-2}\left\|(1-\Delta)^{1 / 2}\left(\gamma_{0, N_{\mathrm{c}}}-\gamma_{0}\right)\right\|_{\mathfrak{S}_{2}\left(L_{\#}^{2}\right)}
$$

and

$$
\left|\widetilde{\mathcal{E}_{0, N_{\mathrm{c}}}}-\mathcal{E}_{0}\right| \leq C N_{\mathrm{c}}^{-2}\left|\mathcal{E}_{0, N_{\mathrm{c}}}-\mathcal{E}_{0}\right|
$$

\subsection{Proofs}

In order to prove Theorem 4.1, we first provide in Section 4.2.1 a decomposition of $\gamma_{0}$ based on spectral projection in Lemma 4.2, relying on Lemma 4.1 for a rigorous justification of the contour integral. In Section 4.2.2, we decompose the difference $\gamma_{0}-\widetilde{\gamma_{0, N_{\mathrm{c}}}}$ into three parts in Lemma 4.3, and we then estimate each of these terms in three of the following Lemmas 4.4, 4.6, and 4.7, relying on an intermediary estimate presented in Lemma 4.5, in order to prove estimate (4.1). Finally, in Section 4.2.3, we provide a proof for estimate (4.2).

\subsubsection{Exact density matrix in terms of approximate density matrix}

Lemma 4.1. Let $\Gamma$ be the circle in the complex plane symmetric with respect to the real axis and containing the real numbers $\lambda_{1}^{0}-1$ and $\epsilon_{\mathrm{F}}$. There exists $N_{\mathrm{c}}^{0} \in \mathbb{N}$ such that for all $N_{\mathrm{c}} \geq N_{\mathrm{c}}^{0}$, $\Gamma$ encloses the lowest $N$ eigenvalues of both the operators $\mathcal{H}$ and $\mathcal{H}_{N_{\mathrm{c}}}$ (respectively defined in (2.1) and (3.1)), and none of the higher ones.

Proof. As $\mathcal{H}$ and $\mathcal{H}_{N_{\mathrm{c}}}$ are self-adjoint operators, their eigenvalues noted respectively $\left(\lambda_{i}^{0}\right)_{i \in \mathbb{N}^{*}}$ and $\left(\lambda_{i, N_{\mathrm{c}}}\right)_{i \in \mathbb{N}^{*}}$ (with increasing values and counting multiplicities) are real. From the gap assumption 2.1, and the definition of the Fermi level, we have

$$
\forall i=1, \ldots, N, \quad \lambda_{i}^{0}<\epsilon_{\mathrm{F}}, \quad \text { and } \quad \forall i>N, \quad \lambda_{i}^{0}>\epsilon_{\mathrm{F}} .
$$


The plane-wave discretization being variational, there holds

$$
\forall i=1, \ldots, \operatorname{dim}\left(X_{N_{\mathrm{c}}}\right), \quad \lambda_{i}^{0} \leq \lambda_{i, N_{\mathrm{c}}}
$$

and the sequence $\left(\lambda_{i, N_{\mathrm{c}}}\right)_{N_{\mathrm{c}}}$ is decreasing. Moreover, classical convergence results (see e.g. [8, Chapter 5]) guarantee that

$$
\max _{i=1, \ldots, N}\left|\lambda_{i, N_{\mathrm{c}}}-\lambda_{i}^{0}\right| \underset{N_{\mathrm{c}} \rightarrow+\infty}{\longrightarrow} 0 .
$$

Therefore, there exists $N_{\mathrm{c}}^{0} \in \mathbb{N}$ such that for all $N_{\mathrm{c}} \geq N_{\mathrm{c}}^{0}, \lambda_{N, N_{\mathrm{c}}} \leq \lambda_{N, N_{\mathrm{c}}^{0}}<\epsilon_{\mathrm{F}}$, and the eigenvalues of the Laplace operator on $X_{N_{\mathrm{c}}}^{\perp}$ are larger than $\lambda_{N+1}^{0}>\epsilon_{\mathrm{F}}$, so that

$$
\forall N_{\mathrm{c}} \geq N_{\mathrm{c}}^{0}, \quad \forall i=1, \ldots, N, \quad \lambda_{i, N_{\mathrm{c}}} \leq \lambda_{N, N_{\mathrm{c}}^{\mathrm{c}}}<\epsilon_{\mathrm{F}}, \quad \text { and } \quad \forall i>N, \quad \lambda_{i, N_{\mathrm{c}}} \geq \lambda_{N+1}^{0}>\epsilon_{\mathrm{F}} .
$$

Combining (4.3) and (4.4) concludes the proof of the lemma.

Using the Cauchy residue theorem and functional calculus for self-adjoint operators, the ground-state density matrix of $\mathcal{H}$ can be written as

$$
\gamma_{0}=\frac{1}{2 \pi i} \oint_{\Gamma}(z-\mathcal{H})^{-1} d z=\frac{1}{2 \pi i} \oint_{\Gamma}\left(z-\mathcal{H}_{N_{\mathrm{c}}}-\mathcal{V}_{N_{\mathrm{c}}}^{\perp}\right)^{-1} d z
$$

Since $\mathcal{V} \in L_{\#}^{\infty}(\Omega), \mathcal{V}_{N_{\mathrm{c}}}^{\perp}$ is $\mathcal{H}_{N_{\mathrm{c}}}$-bounded, and we can perform a Dyson expansion of (4.5) at second order. We obtain

$$
\begin{aligned}
\gamma_{0}= & \frac{1}{2 \pi i} \oint_{\Gamma}\left(z-\mathcal{H}_{N_{\mathrm{c}}}\right)^{-1} d z+\frac{1}{2 \pi i} \oint_{\Gamma}\left(z-\mathcal{H}_{N_{\mathrm{c}}}\right)^{-1} \mathcal{V}_{N_{\mathrm{c}}}^{\perp}\left(z-\mathcal{H}_{N_{\mathrm{c}}}\right)^{-1} d z \\
& +\frac{1}{2 \pi i} \oint_{\Gamma}(z-\mathcal{H})^{-1} \mathcal{V}_{N_{\mathrm{c}}}^{\perp}\left(z-\mathcal{H}_{N_{\mathrm{c}}}\right)^{-1} \mathcal{V}_{N_{\mathrm{c}}}^{\perp}\left(z-\mathcal{H}_{N_{\mathrm{c}}}\right)^{-1} d z
\end{aligned}
$$

where each term of the right-hand side is well-defined.

Lemma 4.2 (Second order expansion of $\gamma_{0}$ ). There holds

$$
\gamma_{0}=\gamma_{0, N_{\mathrm{c}}}+\gamma_{N_{\mathrm{c}}}^{(1)}+\widetilde{Q_{N_{\mathrm{c}}}}
$$

where $\gamma_{N_{\mathrm{c}}}^{(1)}$ is the finite-rank operator defined in (3.9) and where

$$
\widetilde{Q_{N_{\mathrm{c}}}}:=\frac{1}{2 i \pi} \oint_{\Gamma}(z-\mathcal{H})^{-1} \mathcal{V}_{N_{\mathrm{c}}}^{\perp}\left(z-\mathcal{H}_{N_{\mathrm{c}}}\right)^{-1} \mathcal{V}_{N_{\mathrm{c}}}^{\perp}\left(z-\mathcal{H}_{N_{\mathrm{c}}}\right)^{-1} d z
$$

Proof. The operator $\mathcal{H}_{N_{\mathrm{c}}}$ being self-adjoint with compact resolvent, it can be diagonalized in an orthonormal basis. Hence, there exists a sequence $\left(\psi_{k}, \varepsilon_{k}\right)_{k \geq 1}$ with $\left(\psi_{k}\right)_{k \geq 1}$ an orthonormal basis of $L_{\#}^{2}(\Omega)$ consisting of functions of $H_{\#}^{2}(\Omega)$ and $\left(\varepsilon_{k}\right)_{k \geq 1}$ a non-decreasing sequence of real numbers such that

$$
\forall k \geq 1, \quad \mathcal{H}_{N_{\mathrm{c}}} \psi_{k}=\varepsilon_{k} \psi_{k} .
$$

Without loss of generality, we can choose a basis such that, in addition, for $k=1, \ldots, N, \psi_{k}=\phi_{k, N_{c}}$ and $\varepsilon_{k}=\lambda_{k, N_{\mathrm{c}}}$. The operator $\mathcal{H}_{N_{\mathrm{c}}}$ can then be written as

$$
\mathcal{H}_{N_{\mathrm{c}}}=\sum_{k \geq 1} \varepsilon_{k}\left|\psi_{k}\right\rangle\left\langle\psi_{k}\right|
$$

Let us show that the expansions (4.6) and (4.7) are identical. First, we have

$$
\gamma_{0, N_{\mathrm{c}}}=\frac{1}{2 \pi i} \oint_{\Gamma}\left(z-\mathcal{H}_{N_{\mathrm{c}}}\right)^{-1} d z .
$$


Let us now prove that the second term in the right hand side, that is

$$
\gamma^{(1)}:=\frac{1}{2 \pi i} \oint_{\Gamma}\left(z-\mathcal{H}_{N_{\mathrm{c}}}\right)^{-1} \mathcal{V}_{N_{\mathrm{c}}}^{\perp}\left(z-\mathcal{H}_{N_{\mathrm{c}}}\right)^{-1} d z
$$

is in fact equal to the operator $\gamma_{N_{\mathrm{c}}}^{(1)}$ defined in (3.9). We have

$$
\forall k, l \in \mathbb{N}^{*}, \quad\left\langle\psi_{k}\left|\gamma_{N_{\mathrm{c}}}^{(1)}\right| \psi_{l}\right\rangle=\sum_{j=1}^{N}\left\langle\psi_{k} \mid \phi_{j, N_{\mathrm{c}}}^{(1)}\right\rangle\left\langle\phi_{j, N_{\mathrm{c}}} \mid \psi_{l}\right\rangle+\sum_{j=1}^{N}\left\langle\psi_{k} \mid \phi_{j, N_{\mathrm{c}}}\right\rangle\left\langle\phi_{j, N_{\mathrm{c}}}^{(1)} \mid \psi_{l}\right\rangle .
$$

As for all $j=1, \ldots, N, \phi_{j, N_{\mathrm{c}}} \in X_{N_{\mathrm{c}}}$ and $\phi_{j, N_{\mathrm{c}}}^{(1)} \in X_{N_{\mathrm{c}}}^{\perp}$, we have $\left\langle\psi_{k}\left|\gamma_{N_{\mathrm{c}}}^{(1)}\right| \psi_{l}\right\rangle=0$ for all $k, l \in \mathbb{N}^{*}$ such that either $k, l>N$, or $k, l \leq N$. Moreover, for all $k \leq N$ and $l>N$, we have

$$
\begin{aligned}
\left\langle\psi_{k}\left|\gamma_{N_{\mathrm{c}}}^{(1)}\right| \psi_{l}\right\rangle & =\left\langle\phi_{k, N_{\mathrm{c}}}\left|\gamma_{N_{\mathrm{c}}}^{(1)}\right| \psi_{l}\right\rangle=\left\langle\phi_{k, N_{\mathrm{c}}}^{(1)} \mid \psi_{l}\right\rangle=-\left\langle\phi_{k, N_{\mathrm{c}}}\left|\mathcal{V}_{N_{\mathrm{c}}}^{\perp}\left(\mathcal{H}_{N_{\mathrm{c}}}-\lambda_{k, N_{\mathrm{c}}}\right)^{-1}\right| \psi_{l}\right\rangle \\
& =\frac{1}{\lambda_{k, N_{\mathrm{c}}}-\varepsilon_{l}}\left\langle\phi_{k, N_{\mathrm{c}}}\left|\mathcal{V}_{N_{\mathrm{c}}}^{\perp}\right| \psi_{l}\right\rangle=\frac{1}{\varepsilon_{k}-\varepsilon_{l}}\left\langle\psi_{k}\left|\mathcal{V}_{N_{\mathrm{c}}}^{\perp}\right| \psi_{l}\right\rangle,
\end{aligned}
$$

and likewise

$$
\left\langle\psi_{l}\left|\gamma_{N_{\mathrm{c}}}^{(1)}\right| \psi_{k}\right\rangle=\frac{1}{\varepsilon_{l}-\varepsilon_{k}}\left\langle\psi_{l}\left|\mathcal{V}_{N_{\mathrm{c}}}^{\perp}\right| \psi_{k}\right\rangle
$$

Thus, for all $k, l \in \mathbb{N}^{*}$, we have

$$
\left\langle\psi_{k}\left|\gamma_{N_{\mathrm{c}}}^{(1)}\right| \psi_{l}\right\rangle=\frac{1}{\varepsilon_{k}-\varepsilon_{l}}\left(\mathbb{1}_{k \leq N} \mathbb{1}_{l>N}-\mathbb{1}_{k>N} \mathbb{1}_{l \leq N}\right)\left\langle\psi_{k}\left|\mathcal{V}_{N_{\mathrm{c}}}^{\perp}\right| \psi_{l}\right\rangle
$$

On the other hand, for all $k, l \in \mathbb{N}^{*}$, using the Cauchy residue theorem,

$$
\begin{aligned}
\left\langle\psi_{k}\left|\gamma^{(1)}\right| \psi_{l}\right\rangle & =\frac{1}{2 \pi i} \oint_{\Gamma}\left\langle\psi_{k}\left|\left(z-\mathcal{H}_{N_{\mathrm{c}}}\right)^{-1} \mathcal{V}_{N_{\mathrm{c}}}^{\perp}\left(z-\mathcal{H}_{N_{\mathrm{c}}}\right)^{-1}\right| \psi_{l}\right\rangle d z \\
& =\frac{1}{2 \pi i}\left(\oint_{\Gamma}\left(z-\varepsilon_{k}\right)^{-1}\left(z-\varepsilon_{l}\right)^{-1} d z\right)\left\langle\psi_{k}\left|\mathcal{V}_{N_{\mathrm{c}}}^{\perp}\right| \psi_{l}\right\rangle \\
& =\frac{1}{\varepsilon_{k}-\varepsilon_{l}}\left(\mathbb{1}_{k \leq N} \mathbb{1}_{l>N}-\mathbb{1}_{k>N} \mathbb{1}_{l \leq N}\right)\left\langle\psi_{k}\left|\mathcal{V}_{N_{\mathrm{c}}}^{\perp}\right| \psi_{l}\right\rangle
\end{aligned}
$$

Therefore, for all $k, l \in \mathbb{N}^{*},\left\langle\psi_{k}\left|\gamma^{(1)}\right| \psi_{l}\right\rangle=\left\langle\psi_{k}\left|\gamma_{N_{\mathrm{c}}}^{(1)}\right| \psi_{l}\right\rangle$. Finally $\gamma^{(1)}=\gamma_{N_{\mathrm{c}}}^{(1)}$, and the definition of $\widetilde{Q_{N_{\mathrm{c}}}}$ in $(4.8)$ allows one to conclude the proof of the lemma.

\subsubsection{Proof of estimate (4.1)}

Lemma 4.3. There holds

$$
\gamma_{0}-\widetilde{\gamma_{0, N_{\mathrm{c}}}}=\left(\gamma_{0}-\gamma_{0, N_{\mathrm{c}}}\right)^{2}+\widetilde{Q_{N_{\mathrm{c}}}} \gamma_{0, N_{\mathrm{c}}}+\gamma_{0, N_{\mathrm{c}}} \widetilde{Q_{N_{\mathrm{c}}}}
$$

with $\widetilde{Q_{N_{\mathrm{c}}}}$ defined in (4.8).

Proof. Let us first remark, from (4.7), and the property $\gamma_{0, N_{\mathrm{c}}}^{2}=\gamma_{0, N_{\mathrm{c}}}$, that

$$
\begin{aligned}
& \gamma_{0, N_{\mathrm{c}}} \gamma_{0}=\gamma_{0, N_{\mathrm{c}}}+\gamma_{0, N_{\mathrm{c}}} \gamma_{N_{\mathrm{c}}}^{(1)}+\gamma_{0, N_{\mathrm{c}}} \widetilde{Q_{N_{\mathrm{c}}}} \\
& \gamma_{0} \gamma_{0, N_{\mathrm{c}}}=\gamma_{0, N_{\mathrm{c}}}+\gamma_{N_{\mathrm{c}}}^{(1)} \gamma_{0, N_{\mathrm{c}}}+\widetilde{Q_{N_{\mathrm{c}}}} \gamma_{0, N_{\mathrm{c}}} .
\end{aligned}
$$


Moreover, as for all $i, j=1,2, \ldots, N, \phi_{i, N_{\mathrm{c}}} \in X_{N_{\mathrm{c}}}$ and $\phi_{j, N_{\mathrm{c}}}^{(1)} \in X_{N_{\mathrm{c}}}^{\perp}, \phi_{i, N_{\mathrm{c}}}$ is orthogonal to $\phi_{j, N_{\mathrm{c}}}^{(1)}$, and therefore

$$
\begin{aligned}
\gamma_{0, N_{\mathrm{c}}} \gamma_{N_{\mathrm{c}}}^{(1)}+\gamma_{N_{\mathrm{c}}}^{(1)} \gamma_{0, N_{\mathrm{c}}}= & \gamma_{0, N_{\mathrm{c}}}\left(\sum_{i=1}^{N}\left|\phi_{i, N_{\mathrm{c}}}^{(1)}\right\rangle\left\langle\phi_{i, N_{\mathrm{c}}}|+| \phi_{i, N_{\mathrm{c}}}\right\rangle\left\langle\phi_{i, N_{\mathrm{c}}}^{(1)}\right|\right) \\
& +\left(\sum_{i=1}^{N}\left|\phi_{i, N_{\mathrm{c}}}^{(1)}\right\rangle\left\langle\phi_{i, N_{\mathrm{c}}}|+| \phi_{i, N_{\mathrm{c}}}\right\rangle\left\langle\phi_{i, N_{\mathrm{c}}}^{(1)}\right|\right) \gamma_{0, N_{\mathrm{c}}} \\
= & \gamma_{0, N_{\mathrm{c}}} \sum_{i=1}^{N}\left|\phi_{i, N_{\mathrm{c}}}\right\rangle\left\langle\phi_{i, N_{\mathrm{c}}}^{(1)}\left|+\sum_{i=1}^{N}\right| \phi_{i, N_{\mathrm{c}}}^{(1)}\right\rangle\left\langle\phi_{i, N_{\mathrm{c}}}\right| \gamma_{0, N_{\mathrm{c}}} \\
& =\sum_{i=1}^{N}\left|\phi_{i, N_{\mathrm{c}}}\right\rangle\left\langle\phi_{i, N_{\mathrm{c}}}^{(1)}\left|+\sum_{i=1}^{N}\right| \phi_{i, N_{\mathrm{c}}}^{(1)}\right\rangle\left\langle\phi_{i, N_{\mathrm{c}}}\right|=\gamma_{N_{\mathrm{c}}}^{(1)} .
\end{aligned}
$$

Hence

$$
\gamma_{0, N_{\mathrm{c}}} \gamma_{0}+\gamma_{0} \gamma_{0, N_{\mathrm{c}}}=2 \gamma_{0, N_{\mathrm{c}}}+\gamma_{N_{\mathrm{c}}}^{(1)}+\widetilde{Q_{N_{\mathrm{c}}}} \gamma_{0, N_{\mathrm{c}}}+\gamma_{0, N_{\mathrm{c}}} \widetilde{Q_{N_{\mathrm{c}}}}=\gamma_{0, N_{\mathrm{c}}}+\widetilde{\gamma_{0, N_{\mathrm{c}}}}+\widetilde{Q_{N_{\mathrm{c}}}} \gamma_{0, N_{\mathrm{c}}}+\gamma_{0, N_{\mathrm{c}}} \widetilde{Q_{N_{\mathrm{c}}}}
$$

so that

$$
\left(\gamma_{0}-\gamma_{0, N_{\mathrm{c}}}\right)^{2}=\gamma_{0}-\left(\gamma_{0, N_{\mathrm{c}}} \gamma_{0}+\gamma_{0} \gamma_{0, N_{\mathrm{c}}}\right)+\gamma_{0, N_{\mathrm{c}}}=\gamma_{0}-\widetilde{\gamma_{0, N_{\mathrm{c}}}}-\widetilde{Q_{N_{\mathrm{c}}}} \gamma_{0, N_{\mathrm{c}}}-\gamma_{0, N_{\mathrm{c}}} \widetilde{Q_{N_{\mathrm{c}}}},
$$

from which we deduce (4.10).

Lemma 4.4. There exist $C \in \mathbb{R}_{+}$and $N_{\mathrm{c}}^{0} \in \mathbb{N}$, such that for all $N_{\mathrm{c}} \geq N_{\mathrm{c}}^{0}$,

$$
\left\|(1-\Delta)^{1 / 2}\left(\gamma_{0}-\gamma_{0, N_{\mathrm{c}}}\right)^{2}\right\|_{\mathfrak{S}_{2}\left(L_{\#}^{2}\right)} \leq C N_{\mathrm{c}}^{-2}\left\|(1-\Delta)^{1 / 2}\left(\gamma_{0}-\gamma_{0, N_{\mathrm{c}}}\right)\right\|_{\mathfrak{S}_{2}\left(L_{\#}^{2}\right)} .
$$

Proof. By cyclicity of the trace, noting that $\left(\gamma_{0}-\gamma_{0, N_{\mathrm{c}}}\right)$ is of finite rank, and using (2.13) and (2.12),

$$
\begin{aligned}
\left\|(1-\Delta)^{1 / 2}\left(\gamma_{0}-\gamma_{0, N_{\mathrm{c}}}\right)^{2}\right\|_{\mathfrak{S}_{2}\left(L_{\#}^{2}\right)}^{2} & =\operatorname{Tr}\left(\left(\gamma_{0}-\gamma_{0, N_{\mathrm{c}}}\right)^{2}(1-\Delta)\left(\gamma_{0}-\gamma_{0, N_{\mathrm{c}}}\right)^{2}\right) \\
& =\operatorname{Tr}\left(\left(\gamma_{0}-\gamma_{0, N_{\mathrm{c}}}\right)^{2}\left(\gamma_{0}-\gamma_{0, N_{\mathrm{c}}}\right)(1-\Delta)\left(\gamma_{0}-\gamma_{0, N_{\mathrm{c}}}\right)\right) \\
& \leq\left\|\left(\gamma_{0}-\gamma_{0, N_{\mathrm{c}}}\right)^{2}\left(\gamma_{0}-\gamma_{0, N_{\mathrm{c}}}\right)(1-\Delta)\left(\gamma_{0}-\gamma_{0, N_{\mathrm{c}}}\right)\right\|_{\mathfrak{S}_{1}\left(L_{\#}^{2}\right)} \\
& \leq\left\|\gamma_{0}-\gamma_{0, N_{\mathrm{c}}}\right\|^{2}\left\|\left(\gamma_{0}-\gamma_{0, N_{\mathrm{c}}}\right)(1-\Delta)\left(\gamma_{0}-\gamma_{0, N_{\mathrm{c}}}\right)\right\|_{\mathfrak{S}_{1}\left(L_{\#}^{2}\right)} \\
& \leq\left\|\gamma_{0}-\gamma_{0, N_{\mathrm{c}}}\right\|_{\mathfrak{S}_{2}\left(L_{\#}^{2}\right)}^{2}\left\|(1-\Delta)^{1 / 2}\left(\gamma_{0}-\gamma_{0, N_{\mathrm{c}}}\right)\right\|_{\mathfrak{S}_{2}\left(L_{\#}^{2}\right)}^{2} .
\end{aligned}
$$

Using the a priori estimate of $\left\|\gamma_{0}-\gamma_{0, N_{\mathrm{c}}}\right\|_{\mathfrak{S}_{2}\left(L_{\#}^{2}\right)}$ given in (2.26) finishes the proof.

We now provide an estimate for $\left\|(1-\Delta)^{-1 / 2} \mathcal{V}_{N_{\mathrm{c}}}^{\perp} \gamma_{0, N_{\mathrm{c}}}\right\|_{\mathfrak{S}_{2}\left(L_{\#}^{2}\right)}$ which will be useful in the proof of Lemma 4.6 and estimate (4.2).

Lemma 4.5. There exist $C \in \mathbb{R}_{+}$and $N_{\mathrm{c}}^{0} \in \mathbb{N}$, such that for $N_{\mathrm{c}} \geq N_{\mathrm{c}}^{0}$,

$$
\left\|(1-\Delta)^{-1 / 2} \mathcal{V}_{N_{\mathrm{c}}}^{\perp} \gamma_{0, N_{\mathrm{c}}}\right\|_{\mathfrak{S}_{2}\left(L_{\#}^{2}\right)} \leq C\left\|(1-\Delta)^{1 / 2}\left(\gamma_{0}-\gamma_{0, N_{\mathrm{c}}}\right)\right\|_{\mathfrak{S}_{2}\left(L_{\#}^{2}\right)} .
$$

Proof. Decomposing $\mathcal{V}_{N_{\mathrm{c}}}^{\perp} \gamma_{0, N_{\mathrm{c}}}$ as

$$
\mathcal{V}_{N_{\mathrm{c}}}^{\perp} \gamma_{0, N_{\mathrm{c}}}=\mathcal{H}\left(\gamma_{0, N_{\mathrm{c}}}-\gamma_{0}\right)+\mathcal{H} \gamma_{0}-\mathcal{H}_{N_{\mathrm{c}}} \gamma_{0, N_{\mathrm{c}}}
$$

we get

$$
\begin{aligned}
\left\|(1-\Delta)^{-1 / 2} \mathcal{V}_{N_{\mathrm{c}}}^{\perp} \gamma_{0, N_{\mathrm{c}}}\right\|_{\mathfrak{S}_{2}\left(L_{\#}^{2}\right) \leq} & \left\|(1-\Delta)^{-1 / 2} \mathcal{H}\left(\gamma_{0, N_{\mathrm{c}}}-\gamma_{0}\right)\right\|_{\mathfrak{S}_{2}\left(L_{\#}^{2}\right)} \\
& +\left\|(1-\Delta)^{-1 / 2}\left(\mathcal{H} \gamma_{0}-\mathcal{H}_{N_{\mathrm{c}}} \gamma_{0, N_{\mathrm{c}}}\right)\right\|_{\mathfrak{S}_{2}\left(L_{\#}^{2}\right)} .
\end{aligned}
$$


Since $(1-\Delta)^{-1 / 2} \mathcal{H}(1-\Delta)^{-1 / 2}$ is a bounded operator (see e.g. [5, Lemma 1] for a proof of this classical result) and from (2.14), there exists $C \in \mathbb{R}_{+}$such that for all $N_{\mathrm{c}} \in \mathbb{N}$,

$$
\left\|(1-\Delta)^{-1 / 2} \mathcal{H}\left(\gamma_{0, N_{\mathrm{c}}}-\gamma_{0}\right)\right\|_{\mathfrak{S}_{2}\left(L_{\#}^{2}\right)} \leq C\left\|(1-\Delta)^{1 / 2}\left(\gamma_{0, N_{\mathrm{c}}}-\gamma_{0}\right)\right\|_{\mathfrak{S}_{2}\left(L_{\#}^{2}\right)} .
$$

In order to bound the second term of the right-hand side of (4.12), we first rewrite the operator $\mathcal{H} \gamma_{0}-$ $\mathcal{H}_{N_{\mathrm{c}}} \gamma_{0, N_{\mathrm{c}}}$ as follows, denoting by $\lambda_{i j}^{0}=\lambda_{i}^{0} \delta_{i j}$, and using $\lambda_{i j, N_{\mathrm{c}}}^{0}$ defined in (2.23):

$$
\begin{aligned}
\mathcal{H} \gamma_{0}-\mathcal{H}_{N_{\mathrm{c}}} \gamma_{0, N_{\mathrm{c}}} & =\sum_{i=1}^{N} \lambda_{i}^{0}\left|\phi_{i}^{0}\right\rangle\left\langle\phi_{i}^{0}\left|-\sum_{i, j=1}^{N} \lambda_{i j, N_{\mathrm{c}}}^{0}\right| \phi_{i, N_{\mathrm{c}}}^{0}\right\rangle\left\langle\phi_{j, N_{\mathrm{c}}}^{0}\right| \\
& =\sum_{i=1}^{N} \lambda_{i}^{0}\left(\left|\phi_{i}^{0}\right\rangle\left\langle\phi_{i}^{0}|-| \phi_{i, N_{\mathrm{c}}}^{0}\right\rangle\left\langle\phi_{i, N_{\mathrm{c}}}^{0}\right|\right)+\sum_{i=1}^{N} \lambda_{i}^{0}\left|\phi_{i, N_{\mathrm{c}}}^{0}\right\rangle\left\langle\phi_{i, N_{\mathrm{c}}}^{0}\left|-\sum_{i, j=1}^{N} \lambda_{i j, N_{\mathrm{c}}}^{0}\right| \phi_{i, N_{\mathrm{c}}}^{0}\right\rangle\left\langle\phi_{j, N_{\mathrm{c}}}^{0}\right| \\
& =\sum_{i=1}^{N} \lambda_{i}^{0}\left(\left|\phi_{i}^{0}\right\rangle\left\langle\phi_{i}^{0}|-| \phi_{i, N_{\mathrm{c}}}^{0}\right\rangle\left\langle\phi_{i, N_{\mathrm{c}}}^{0}\right|\right)+\sum_{i, j=1}^{N}\left(\lambda_{i j}^{0}-\lambda_{i j, N_{\mathrm{c}}}^{0}\right)\left|\phi_{i, N_{\mathrm{c}}}^{0}\right\rangle\left\langle\phi_{j, N_{\mathrm{c}}}^{0}\right| .
\end{aligned}
$$

Using the triangle and the Cauchy-Schwarz inequality, we get

$$
\begin{aligned}
\left.\left\|\mathcal{H} \gamma_{0}-\mathcal{H}_{N_{\mathrm{c}}} \gamma_{0, N_{\mathrm{c}}}\right\|\right|_{\mathfrak{S}_{2}\left(L_{\#}^{2}\right) \leq} & \left\|\sum_{i=1}^{N} \lambda_{i}^{0}\left(\left|\phi_{i}^{0}\right\rangle\left\langle\phi_{i}^{0}|-| \phi_{i, N_{\mathrm{c}}}^{0}\right\rangle\left\langle\phi_{i, N_{\mathrm{c}}}^{0}\right|\right)\right\|_{\mathfrak{S}_{2}\left(L_{\#}^{2}\right)} \\
& +\| \sum_{i, j=1}^{N}\left(\lambda_{i j}^{0}-\lambda_{i j, N_{\mathrm{c}}}^{0}\right)\left|\phi_{i, N_{\mathrm{c}}}^{0}\right\rangle\left\langle\phi_{j, N_{\mathrm{c}}}^{0}\right| \|_{\mathfrak{S}_{2}\left(L_{\#}^{2}\right)} \\
\leq & \sum_{i=1}^{N}\left|\lambda_{i}^{0}\right|\left\|\left(\left|\phi_{i}^{0}\right\rangle\left\langle\phi_{i}^{0}|-| \phi_{i, N_{\mathrm{c}}}^{0}\right\rangle\left\langle\phi_{i, N_{\mathrm{c}}}^{0}\right|\right)\right\|_{\mathfrak{S}_{2}\left(L_{\#}^{2}\right)} \\
& +\sum_{i, j=1}^{N}\left|\lambda_{i j}^{0}-\lambda_{i j, N_{\mathrm{c}}}^{0}\right| \|\left|\phi_{i, N_{\mathrm{c}}}^{0}\right\rangle\left\langle\phi_{j, N_{\mathrm{c}}}^{0}\right| \|_{\mathfrak{S}_{2}\left(L_{\#}^{2}\right)} \\
\leq & \left(\sum_{i=1}^{N}\left|\lambda_{i}^{0}\right|^{2}\right)^{1 / 2}\left(\sum_{i=1}^{N} \|\left|\phi_{i}^{0}\right\rangle\left\langle\phi_{i}^{0}|-| \phi_{i, N_{\mathrm{c}}}^{0}\right\rangle\left\langle\phi_{i, N_{\mathrm{c}}}^{0}\right| \|_{\mathfrak{S}_{2}\left(L_{\#}^{2}\right)}^{2}\right)^{1 / 2} \\
& +\left\|\Lambda^{0}-\Lambda_{N_{\mathrm{c}}}^{0}\right\|_{F}\left(\sum_{i, j=1}^{N} \|\left|\phi_{i, N_{\mathrm{c}}}^{0}\right\rangle\left\langle\phi_{j, N_{\mathrm{c}}}^{0} \|_{\mathfrak{S}_{2}\left(L_{\#}^{2}\right)}^{2}\right)^{1 / 2}\right. \\
\leq & 2\left(\sum_{i=1}^{N}\left|\lambda_{i}^{0}\right|^{2}\right)^{1 / 2}\left\|\Phi^{0}-\Phi_{N_{\mathrm{c}}}^{0}\right\|_{L_{\#}^{2}}+N\left\|\Lambda^{0}-\Lambda_{N_{\mathrm{c}}}^{0}\right\|_{F} .
\end{aligned}
$$

Using (2.24), (2.27), and (2.28), we obtain that there exist $C \in \mathbb{R}_{+}$and $N_{\mathrm{c}}^{0} \in \mathbb{N}$ such that for all $N_{\mathrm{c}} \geq N_{\mathrm{c}}^{0}$,

$$
\left\|\mathcal{H} \gamma_{0}-\mathcal{H}_{N_{\mathrm{c}}} \gamma_{0, N_{\mathrm{c}}}\right\|_{\mathfrak{S}_{2}\left(L_{\#}^{2}\right)} \leq C N_{\mathrm{c}}^{-1}\left\|(1-\Delta)^{1 / 2}\left(\gamma_{0}-\gamma_{0, N_{\mathrm{c}}}\right)\right\|_{\mathfrak{S}_{2}\left(L_{\#}^{2}\right)}+C\left\|(1-\Delta)^{1 / 2}\left(\gamma_{0}-\gamma_{0, N_{\mathrm{c}}}\right)\right\|_{\mathfrak{S}_{2}\left(L_{\#}^{2}\right)}^{2} .
$$

Since $\left\|(1-\Delta)^{-1 / 2}\right\| \leq 1$, this shows in particular that

$$
\left\|(1-\Delta)^{-1 / 2}\left(\mathcal{H} \gamma_{0}-\mathcal{H}_{N_{\mathrm{c}}} \gamma_{0, N_{\mathrm{c}}}\right)\right\|_{\mathfrak{S}_{2}\left(L_{\#}^{2}\right)} \leq C\left\|(1-\Delta)^{1 / 2}\left(\gamma_{0}-\gamma_{0, N_{\mathrm{c}}}\right)\right\|_{\mathfrak{S}_{2}\left(L_{\#}^{2}\right)} .
$$

Inserting (4.13) and (4.14) in (4.12) concludes the proof of the lemma. 
Lemma 4.6. There exist $C \in \mathbb{R}_{+}$and $N_{\mathrm{c}}^{0} \in \mathbb{N}$ such that for all $N_{\mathrm{c}} \geq N_{\mathrm{c}}^{0}$,

$$
\left\|(1-\Delta)^{1 / 2} \widetilde{Q_{N_{\mathrm{c}}}} \gamma_{0, N_{\mathrm{c}}}\right\|_{\mathfrak{S}_{2}\left(L_{\#}^{2}\right)} \leq C N_{\mathrm{c}}^{-2}\left\|(1-\Delta)^{1 / 2}\left(\gamma_{0}-\gamma_{0, N_{\mathrm{c}}}\right)\right\|_{\mathfrak{S}_{2}\left(L_{\#}^{2}\right)} .
$$

Proof. Using the definition (4.8) and the fact that $\left(z-\mathcal{H}_{N_{\mathrm{c}}}\right)^{-1}$ and $\gamma_{0, N_{\mathrm{c}}}$ commute, we obtain

$$
(1-\Delta)^{1 / 2} \widetilde{Q_{N_{\mathrm{c}}}} \gamma_{0, N_{\mathrm{c}}}=\frac{1}{2 i \pi} \oint_{\Gamma}(1-\Delta)^{1 / 2}(z-\mathcal{H})^{-1} \mathcal{V}_{N_{\mathrm{c}}}^{\perp}\left(z-\mathcal{H}_{N_{\mathrm{c}}}\right)^{-1} \mathcal{V}_{N_{\mathrm{c}}}^{\perp} \gamma_{0, N_{\mathrm{c}}}\left(z-\mathcal{H}_{N_{\mathrm{c}}}\right)^{-1} d z
$$

Since $\operatorname{Ran}\left(\mathcal{V}_{N_{\mathrm{c}}}^{\perp} \gamma_{0, N_{\mathrm{c}}}\right) \subset X_{N_{\mathrm{c}}}^{\perp}$, we have $\mathcal{V}_{N_{\mathrm{c}}}^{\perp} \gamma_{0, N_{\mathrm{c}}}=\Pi_{N_{\mathrm{c}}}^{\perp} \mathcal{V}_{N_{\mathrm{c}}}^{\perp} \gamma_{0, N_{\mathrm{c}}}$. Observing that

$$
\left(z-\mathcal{H}_{N_{\mathrm{c}}}\right)^{-1} \Pi_{N_{\mathrm{c}}}^{\perp}=\Pi_{N_{\mathrm{c}}}^{\perp}\left(z+\frac{1}{2} \Delta_{\mid X_{N_{\mathrm{c}}}}\right)^{-1} \Pi_{N_{\mathrm{c}}}^{\perp}
$$

we thus obtain

$$
\left(z-\mathcal{H}_{N_{\mathrm{c}}}\right)^{-1} \mathcal{V}_{N_{\mathrm{c}}}^{\perp} \gamma_{0, N_{\mathrm{c}}}=\left(z-\mathcal{H}_{N_{\mathrm{c}}}\right)^{-1} \Pi_{N_{\mathrm{c}}}^{\perp} \mathcal{V}_{N_{\mathrm{c}}}^{\perp} \gamma_{0, N_{\mathrm{c}}}=\Pi_{N_{\mathrm{c}}}^{\perp}\left(z+\frac{1}{2} \Delta_{\mid X_{N_{\mathrm{c}}}^{\perp}}\right)^{-1} \mathcal{V}_{N_{\mathrm{c}}}^{\perp} \gamma_{0, N_{\mathrm{c}}}
$$

Therefore,

$$
\begin{aligned}
(1-\Delta)^{1 / 2} \widetilde{Q_{N_{\mathrm{c}}}} \gamma_{0, N_{\mathrm{c}}}=\frac{1}{2 i \pi} \oint_{\Gamma} & {\left[(1-\Delta)^{1 / 2}(z-\mathcal{H})^{-1}(1-\Delta)^{1 / 2}\right]\left[(1-\Delta)^{-1 / 2} \mathcal{V}_{N_{\mathrm{c}}}^{\perp}(1-\Delta)^{-1 / 2} \Pi_{N_{\mathrm{c}}}^{\perp}\right] } \\
\times & {\left[\Pi_{N_{\mathrm{c}}}^{\perp}(1-\Delta)^{1 / 2}\left(z+\frac{1}{2} \Delta\right)^{-1}(1-\Delta)^{1 / 2} \Pi_{N_{\mathrm{c}}}^{\perp}\right] } \\
\times & {\left[(1-\Delta)^{-1 / 2} \mathcal{V}_{N_{\mathrm{c}}}^{\perp} \gamma_{0, N_{\mathrm{c}}}\left(z-\mathcal{H}_{N_{\mathrm{c}}}\right)^{-1}\right] d z . }
\end{aligned}
$$

First,

$$
\begin{aligned}
\left\|(1-\Delta)^{-1 / 2} \mathcal{V}_{N_{\mathrm{c}}}^{\perp}(1-\Delta)^{-1 / 2} \Pi_{N_{\mathrm{c}}}^{\perp}\right\| & =\left\|(1-\Delta)^{-1 / 2} \mathcal{V}(1-\Delta)^{-1 / 2} \Pi_{N_{\mathrm{c}}}^{\perp}\right\| \\
& =\left\|\Pi_{N_{\mathrm{c}}}^{\perp}(1-\Delta)^{-1 / 2} \mathcal{V}(1-\Delta)^{-1 / 2}\right\| \\
& \leq\left\|\Pi_{N_{\mathrm{c}}}^{\perp}(1-\Delta)^{-1}\right\|\left\|(1-\Delta)^{1 / 2} \mathcal{V}(1-\Delta)^{-1 / 2}\right\|
\end{aligned}
$$

Since $\left\|(1-\Delta)^{1 / 2} \mathcal{V}(1-\Delta)^{-1 / 2}\right\|$ equals the operator norm of $\mathcal{V}$, considered as a multiplicative operator from $H_{\#}^{1}(\Omega)$ to $H_{\#}^{1}(\Omega)$, it can be shown using classical Sobolev embeddings that there exists $C \in \mathbb{R}_{+}$such that

$$
\left\|(1-\Delta)^{1 / 2} \mathcal{V}(1-\Delta)^{-1 / 2}\right\| \leq C\left(\|\mathcal{V}\|_{L^{\infty}}+\|\nabla \mathcal{V}\|_{L^{3}}\right),
$$

which is bounded under Assumption 2.2. Since $\left\|\Pi_{N_{\mathrm{c}}}^{\perp}(1-\Delta)^{-1}\right\| \leq\left(1+N_{\mathrm{c}}^{2}\right)^{-1}$ for all $N_{\mathrm{c}} \in \mathbb{N}$, there exist $C \in \mathbb{R}_{+}$and $N_{\mathrm{c}}^{0} \in \mathbb{N}$ such that for all $N_{\mathrm{c}} \geq N_{\mathrm{c}}^{0}$,

$$
\left\|(1-\Delta)^{-1 / 2} \mathcal{V}_{N_{\mathrm{c}}}^{\perp}(1-\Delta)^{-1 / 2} \Pi_{N_{\mathrm{c}}}^{\perp}\right\| \leq C N_{\mathrm{c}}^{-2} .
$$

Finally,

$$
\begin{aligned}
\max _{z \in \Gamma}\left\|(1-\Delta)^{-1 / 2} \mathcal{V}_{N_{\mathrm{c}}}^{\perp} \gamma_{0, N_{\mathrm{c}}}\left(z-\mathcal{H}_{N_{\mathrm{c}}}\right)^{-1}\right\|_{\mathfrak{S}_{2}\left(L_{\#}^{2}\right)} & =\left(\sum_{i=1}^{N} \max _{z \in \Gamma}\left|z-\lambda_{i, N_{\mathrm{c}}}\right|^{-2}\left\|(1-\Delta)^{-1 / 2} \mathcal{V}_{N_{\mathrm{c}}}^{\perp} \phi_{i, N_{\mathrm{c}}}\right\|_{L_{\#}^{2}}^{2}\right)^{1 / 2} \\
& \leq \max _{\substack{z \in \Gamma, i=1, \ldots, N}}\left|z-\lambda_{i, N_{\mathrm{c}}}\right|^{-2}\left(\sum_{i=1}^{N}\left\|(1-\Delta)^{-1 / 2} \mathcal{V}_{N_{\mathrm{c}}}^{\perp} \phi_{i, N_{\mathrm{c}}}\right\|_{L_{\#}^{2}}^{2}\right)^{1 / 2} \\
& =\max _{\substack{z \in \Gamma, i=1, \ldots, N}}\left|z-\lambda_{i, N_{\mathrm{c}}}\right|^{-2}\left\|(1-\Delta)^{-1 / 2} \mathcal{V}_{N_{\mathrm{c}}}^{\perp} \gamma_{0, N_{\mathrm{c}}}\right\|_{\mathfrak{S}_{2}\left(L_{\#}^{2}\right) .} .
\end{aligned}
$$


From the definition of the contour $\Gamma, \max _{\substack{z \in \Gamma, i=1, \ldots, N}}\left|z-\lambda_{i, N_{\mathrm{c}}}\right|^{-2}$ is bounded uniformly in $N_{\mathrm{c}}$ for $N_{\mathrm{c}}$ large enough. Hence, combining the above inequality with (4.11), we obtain that there exist $C \in \mathbb{R}_{+}$and $N_{\mathrm{c}}^{0} \in \mathbb{N}$ such that for all $N_{\mathrm{c}} \geq N_{\mathrm{c}}^{0}$,

$$
\max _{z \in \Gamma}\left\|(1-\Delta)^{-1 / 2} \mathcal{V}_{N_{\mathrm{c}}}^{\perp} \gamma_{0, N_{\mathrm{c}}}\left(z-\mathcal{H}_{N_{\mathrm{c}}}\right)^{-1}\right\|_{\mathfrak{S}_{2}\left(L_{\#}^{2}\right)} \leq C\left\|(1-\Delta)^{1 / 2}\left(\gamma_{0, N_{\mathrm{c}}}-\gamma_{0}\right)\right\|_{\mathfrak{S}_{2}\left(L_{\#}^{2}\right)} .
$$

We are now in position to estimate $\left\|(1-\Delta)^{1 / 2} \widetilde{Q_{N_{\mathrm{c}}}} \gamma_{0, N_{\mathrm{c}}}\right\|_{\mathfrak{S}_{2}\left(L_{\#}^{2}\right)}$. We start from (4.15). It is classical that $\max _{z \in \Gamma}\left\|(1-\Delta)^{1 / 2}(z-\mathcal{H})^{-1}(1-\Delta)^{1 / 2}\right\|$ is bounded (see e.g. [5, Lemma 1]). Moreover, $\max _{z \in \Gamma} \| \Pi_{N_{\mathrm{c}}}^{\perp}(1-\Delta)^{1 / 2}(z+$ $\left.\frac{1}{2} \Delta\right)^{-1}(1-\Delta)^{1 / 2} \Pi_{N_{\mathrm{c}}}^{\perp} \|$ is also bounded. Using estimates (4.16) and (4.17) allows one to conclude the proof of the lemma.

Lemma 4.7. There exist $C \in \mathbb{R}_{+}$and $N_{\mathrm{c}}^{0} \in \mathbb{N}$ such that for all $N_{\mathrm{c}} \geq N_{\mathrm{c}}^{0}$,

$$
\left\|(1-\Delta)^{1 / 2} \gamma_{0, N_{\mathrm{c}}} \widetilde{Q_{N_{\mathrm{c}}}}\right\|_{\mathfrak{S}_{2}\left(L_{\#}^{2}\right)} \leq C N_{\mathrm{c}}^{-2}\left\|(1-\Delta)^{1 / 2}\left(\gamma_{0}-\gamma_{0, N_{\mathrm{c}}}\right)\right\|_{\mathfrak{S}_{2}\left(L_{\#}^{2}\right)}
$$

Proof. Noting that $\gamma_{0, N_{\mathrm{c}}}^{2}=\gamma_{0, N_{\mathrm{c}}}$, using (2.15) and the cyclicity of the trace, we obtain

$$
\begin{aligned}
\left\|(1-\Delta)^{1 / 2} \gamma_{0, N_{\mathrm{c}}} \widetilde{Q_{N_{\mathrm{c}}}}\right\|_{\mathfrak{S}_{2}\left(L_{\#}^{2}\right)} & \leq\left\|(1-\Delta)^{1 / 2} \gamma_{0, N_{\mathrm{c}}}\right\|_{\mathfrak{S}_{2}\left(L_{\#}^{2}\right.}\left\|\gamma_{0, N_{\mathrm{c}}} \widetilde{Q_{N_{\mathrm{c}}}}\right\|_{\mathfrak{S}_{2}\left(L_{\#}^{2}\right)} \\
& =\left\|(1-\Delta)^{1 / 2} \gamma_{0, N_{\mathrm{c}}}\right\|_{\mathfrak{S}_{2}\left(L_{\#}^{2}\right.}\left\|\widetilde{Q_{N_{\mathrm{c}}}} \gamma_{0, N_{\mathrm{c}}}\right\|_{\mathfrak{S}_{2}\left(L_{\#}^{2}\right)},
\end{aligned}
$$

since $\gamma_{0, N_{\mathrm{c}}}$ is a finite-rank orthogonal projector. Moreover, as the orbitals $\left(\phi_{i, N_{\mathrm{c}}}\right)_{i=1, \cdots, N}$ are bounded in $H_{\#}^{1}(\Omega)$ uniformly in $N_{\mathrm{c}},\left\|(1-\Delta)^{1 / 2} \gamma_{0, N_{\mathrm{c}}}\right\|_{\mathfrak{S}_{2}\left(L_{\#}^{2}\right)}$ is also bounded uniformly in $N_{\mathrm{c}}$. On top of that, noting that $\left\|(1-\Delta)^{-1 / 2}\right\| \leq 1$, we have

$$
\left\|\widetilde{Q_{N_{\mathrm{c}}}} \gamma_{0, N_{\mathrm{c}}}\right\|_{\mathfrak{S}_{2}\left(L_{\#}^{2}\right)} \leq\left\|(1-\Delta)^{-1 / 2}\right\|\left\|(1-\Delta)^{1 / 2} \widetilde{Q_{N_{\mathrm{c}}}} \gamma_{0, N_{\mathrm{c}}}\right\|_{\mathfrak{S}_{2}\left(L_{\#}^{2}\right)} \leq\left\|(1-\Delta)^{1 / 2} \widetilde{Q_{N_{\mathrm{c}}}} \gamma_{0, N_{\mathrm{c}}}\right\|_{\mathfrak{S}_{2}\left(L_{\#}^{2}\right)}
$$

Therefore, we can use the estimate of Lemma 4.6 to conclude.

From Lemma 4.3, and using the estimates of Lemmas 4.4, 4.6 and 4.7, we easily get estimate (4.1).

\subsubsection{Proof of estimate (4.2)}

If the perturbed density matrix were satisfying $\widetilde{\gamma_{0, N_{\mathrm{c}}}} \in \mathcal{K}$, we could deduce from (2.18) that the error $\widetilde{\mathcal{E}_{0, N_{\mathrm{c}}}}-\mathcal{E}_{0}$ would be non-negative and converge to zero as $\left\|(1-\Delta)^{1 / 2}\left(\widetilde{\gamma_{0, N_{\mathrm{c}}}}-\gamma_{0}\right)\right\|_{\mathfrak{S}_{2}\left(L_{\#}^{2}\right)}^{2}$ when $N_{\mathrm{c}}$ goes to infinity, yielding an improvement factor for the energy of order $N_{\mathrm{c}}^{-4}$. However, as pointed out in Remark 3.1, $\widetilde{\gamma_{0, N_{\mathrm{c}}}}$ does not belong to $\mathcal{K}$ in general. Yet, the perturbed energy, written as a sum of second-order corrected eigenvalues, can be seen as a second-order correction of the discrete energy. Therefore, we are going to show that the improvement factor for the energy is in fact of order $N_{\mathrm{c}}^{-2}$.

We have $\widetilde{\mathcal{E}_{0, N_{\mathrm{c}}}}-\mathcal{E}_{0}=\operatorname{Tr}\left(\gamma_{0, N_{\mathrm{c}}} \mathcal{H} \widetilde{\gamma_{0, N_{\mathrm{c}}}}\right)-\operatorname{Tr}\left(\gamma_{0} \mathcal{H} \gamma_{0}\right)$. As $\operatorname{Tr}\left(\left(\gamma_{0}\right)^{2}\right)=N$ and $\operatorname{Tr}\left(\gamma_{0, N_{\mathrm{c}}} \widetilde{\gamma_{0, N_{\mathrm{c}}}}\right)=$ $\operatorname{Tr}\left(\gamma_{0, N_{\mathrm{c}}}^{2}\right)=N$, the energy difference can be written as follows

$$
\begin{aligned}
& \widetilde{\mathcal{E}_{0, N_{\mathrm{c}}}}-\varepsilon_{0}=\operatorname{Tr}\left(\gamma_{0, N_{\mathrm{c}}}\left(\mathcal{H}-\epsilon_{\mathrm{F}}\right) \widetilde{\gamma_{0, N_{\mathrm{c}}}}\right)-\operatorname{Tr}\left(\gamma_{0}\left(\mathcal{H}-\epsilon_{\mathrm{F}}\right) \gamma_{0}\right) \\
& =\operatorname{Tr}\left(\left(\gamma_{0, N_{\mathrm{c}}}-\gamma_{0}\right)\left(\mathcal{H}-\epsilon_{\mathrm{F}}\right)\left(\widetilde{\gamma_{0, N_{\mathrm{c}}}}-\gamma_{0}\right)\right)+\operatorname{Tr}\left(\gamma_{0}\left(\mathcal{H}-\epsilon_{\mathrm{F}}\right)\left(\widetilde{\gamma_{0, N_{\mathrm{c}}}}+\gamma_{0, N_{\mathrm{c}}}-2 \gamma_{0}\right)\right) \\
& =\operatorname{Tr}\left(\left(\gamma_{0, N_{\mathrm{c}}}-\gamma_{0}\right)\left(\mathcal{H}-\epsilon_{\mathrm{F}}\right)\left(\widetilde{\gamma_{0, N_{\mathrm{c}}}}-\gamma_{0}\right)\right)+2 \operatorname{Tr}\left(\gamma_{0}\left(\mathcal{H}-\epsilon_{\mathrm{F}}\right)\left(\gamma_{0, N_{\mathrm{c}}}-\gamma_{0}\right)\right)+\operatorname{Tr}\left(\gamma_{0}\left(\mathcal{H}-\epsilon_{\mathrm{F}}\right) \gamma_{N_{\mathrm{c}}}^{(1)}\right) \text {. }
\end{aligned}
$$


We now estimate each of these three terms. First, noting that $\left(\mathcal{H}-\epsilon_{\mathrm{F}}\right)=-\gamma_{0}\left|\mathcal{H}-\epsilon_{\mathrm{F}}\right|+\left(1-\gamma_{0}\right)\left|\mathcal{H}-\epsilon_{\mathrm{F}}\right|$, using the triangle and the Cauchy-Schwarz inequalities, the fact that $\left|\mathcal{H}-\epsilon_{\mathrm{F}}\right|, \gamma_{0}$ and $\left(1-\gamma_{0}\right)$ commute, and (2.15), we get

$$
\begin{aligned}
\left|\operatorname{Tr}\left(\left(\gamma_{0, N_{\mathrm{c}}}-\gamma_{0}\right)\left(\mathcal{H}-\epsilon_{\mathrm{F}}\right)\left(\widetilde{\gamma_{0, N_{\mathrm{c}}}}-\gamma_{0}\right)\right)\right|= & \left.\mid \operatorname{Tr}\left(\left(\gamma_{0, N_{\mathrm{c}}}-\gamma_{0}\right)\left(1-\gamma_{0}\right)\left|\mathcal{H}-\epsilon_{\mathrm{F}}\right| \widetilde{\gamma_{0, N_{\mathrm{c}}}}-\gamma_{0}\right)\right) \\
& \left.-\operatorname{Tr}\left(\left(\gamma_{0, N_{\mathrm{c}}}-\gamma_{0}\right) \gamma_{0}\left|\mathcal{H}-\epsilon_{\mathrm{F}}\right| \widetilde{\gamma_{0, N_{\mathrm{c}}}}-\gamma_{0}\right)\right) \mid \\
\leq & \left(\left\|\left|\mathcal{H}-\epsilon_{\mathrm{F}}\right|^{1 / 2}\left(1-\gamma_{0}\right)\left(\gamma_{0, N_{\mathrm{c}}}-\gamma_{0}\right)\right\|_{\mathfrak{S}_{2}\left(L_{\#}^{2}\right)}\right. \\
& \left.+\left\|\left|\mathcal{H}-\epsilon_{\mathrm{F}}\right|^{1 / 2} \gamma_{0}\left(\gamma_{0, N_{\mathrm{c}}}-\gamma_{0}\right)\right\|_{\mathfrak{S}_{2}\left(L_{\#}^{2}\right)}\right)\left\|\left|\mathcal{H}-\epsilon_{\mathrm{F}}\right|^{1 / 2}\left(\widetilde{\gamma_{0, N_{\mathrm{c}}}}-\gamma_{0}\right)\right\|_{\mathfrak{S}_{2}\left(L_{\#}^{2}\right)} \\
\leq & \left.2\left\|\left|\mathcal{H}-\epsilon_{\mathrm{F}}\right|^{1 / 2}\left(\gamma_{0, N_{\mathrm{c}}}-\gamma_{0}\right)\right\|_{\mathfrak{S}_{2}\left(L_{\#}^{2}\right.}\right)\left\|\left|\mathcal{H}-\epsilon_{\mathrm{F}}\right|^{1 / 2}\left(\widetilde{\gamma_{0, N_{\mathrm{c}}}}-\gamma_{0}\right)\right\|_{\mathfrak{S}_{2}\left(L_{\#}^{2}\right)} .
\end{aligned}
$$

From (2.16) and (4.1), there exist $C \in \mathbb{R}_{+}$and $N_{\mathrm{c}}^{0} \in \mathbb{N}$, such that for all $N_{\mathrm{c}} \geq N_{\mathrm{c}}^{0}$,

$$
\left|\operatorname{Tr}\left(\left(\gamma_{0, N_{\mathrm{c}}}-\gamma_{0}\right)\left(\mathcal{H}-\epsilon_{\mathrm{F}}\right)\left(\widetilde{\gamma_{0, N_{\mathrm{c}}}}-\gamma_{0}\right)\right)\right| \leq C N_{\mathrm{c}}^{-2}\left\|(1-\Delta)^{1 / 2}\left(\gamma_{0, N_{\mathrm{c}}}-\gamma_{0}\right)\right\|_{\mathfrak{S}_{2}\left(L_{\#}^{2}\right)}^{2} .
$$

Second, noting that for all $i=1, \ldots, N,\left\langle\phi_{i}^{0}\left|\gamma_{0}-\gamma_{0, N_{\mathrm{c}}}\right| \phi_{i}^{0}\right\rangle \geq 0$, we get

$$
\begin{aligned}
\left|\operatorname{Tr}\left(\gamma_{0}\left(\mathcal{H}-\epsilon_{\mathrm{F}}\right)\left(\gamma_{0, N_{\mathrm{c}}}-\gamma_{0}\right)\right)\right| & =\left|\sum_{i=1}^{N}\left(\lambda_{i}^{0}-\epsilon_{\mathrm{F}}\right)\left\langle\phi_{i}^{0}\left|\gamma_{0, N_{\mathrm{c}}}-\gamma_{0}\right| \phi_{i}^{0}\right\rangle\right| \\
& \leq \max _{i=1, \ldots, N}\left|\lambda_{i}^{0}-\epsilon_{\mathrm{F}}\right| \sum_{i=1}^{N}\left\langle\phi_{i}^{0}\left|\gamma_{0}-\gamma_{0, N_{\mathrm{c}}}\right| \phi_{i}^{0}\right\rangle \\
& =\max _{i=1, \ldots, N}\left|\lambda_{i}^{0}-\epsilon_{\mathrm{F}}\right|\left(N-\operatorname{Tr}\left(\gamma_{0, N_{\mathrm{c}}} \gamma_{0}\right)\right) \\
& =\frac{1}{2} \max _{i=1, \ldots, N}\left|\lambda_{i}^{0}-\epsilon_{\mathrm{F}}\right|\left\|\gamma_{0}-\gamma_{0, N_{\mathrm{c}}}\right\|_{\mathfrak{S}_{2}\left(L_{\#}^{2}\right)}^{2} .
\end{aligned}
$$

From (2.27), there exist $C \in \mathbb{R}_{+}$and $N_{\mathrm{c}}^{0} \in \mathbb{N}$, such that for all $N_{\mathrm{c}} \geq N_{\mathrm{c}}^{0}$,

$$
\left|\operatorname{Tr}\left(\gamma_{0}\left(\mathcal{H}-\epsilon_{\mathrm{F}}\right)\left(\gamma_{0, N_{\mathrm{c}}}-\gamma_{0}\right)\right)\right| \leq C N_{\mathrm{c}}^{-2}\left\|(1-\Delta)^{1 / 2}\left(\gamma_{0}-\gamma_{0, N_{\mathrm{c}}}\right)\right\|_{\mathfrak{S}_{2}\left(L_{\#}^{2}\right)}^{2}
$$

Third, noting that for $i, j=1, \ldots, N,\left\langle\phi_{j, N_{\mathrm{c}}}^{(1)} \mid \phi_{i, N_{\mathrm{c}}}^{0}\right\rangle=0,\left\|\phi_{i}^{0}\right\|_{L_{\#}^{2}}=1,\left\|\phi_{j, N_{\mathrm{c}}}\right\|_{L_{\#}^{2}}=1$, and using (3.9) and the Cauchy-Schwarz inequality, we get

$$
\begin{aligned}
\left|\operatorname{Tr}\left(\gamma_{0}\left(\mathcal{H}-\epsilon_{\mathrm{F}}\right) \gamma_{N_{\mathrm{c}}}^{(1)}\right)\right| & =\left|\sum_{i=1}^{N}\left(\lambda_{i}^{0}-\epsilon_{\mathrm{F}}\right)\left\langle\phi_{i}^{0}\left|\gamma_{N_{\mathrm{c}}}^{(1)}\right| \phi_{i}^{0}\right\rangle\right| \\
& =2\left|\sum_{i=1}^{N} \sum_{j=1}^{N}\left(\lambda_{i}^{0}-\epsilon_{\mathrm{F}}\right)\left\langle\phi_{i}^{0} \mid \phi_{j, N_{\mathrm{c}}}\right\rangle\left\langle\phi_{j, N_{\mathrm{c}}}^{(1)} \mid \phi_{i}^{0}\right\rangle\right| \\
& \leq 2 \max _{i=1, \ldots, N}\left|\lambda_{i}^{0}-\epsilon_{\mathrm{F}}\right| \sum_{i=1}^{N} \sum_{j=1}^{N}\left|\left\langle\phi_{i}^{0} \mid \phi_{j, N_{\mathrm{c}}}\right\rangle\left\langle\phi_{j, N_{\mathrm{c}}}^{(1)} \mid \phi_{i}^{0}-\phi_{i, N_{\mathrm{c}}}^{0}\right\rangle\right| \\
& \leq 2 \max _{i=1, \ldots, N}\left|\lambda_{i}^{0}-\epsilon_{\mathrm{F}}\right| \sum_{i=1}^{N}\left\|\phi_{i}^{0}-\phi_{i, N_{\mathrm{c}}}^{0}\right\|_{L_{\#}^{2}} \sum_{j=1}^{N}\left\|\phi_{j, N_{\mathrm{c}}}^{(1)}\right\|_{L_{\#}^{2}} \\
& \leq 2 N \max _{i=1, \ldots, N}\left|\lambda_{i}^{0}-\epsilon_{\mathrm{F}}\right|\left\|\Phi^{0}-\Phi_{N_{\mathrm{c}}}^{0}\right\|_{L_{\#}^{2}}\left(\sum_{j=1}^{N}\left\|\phi_{j, N_{\mathrm{c}}}^{(1)}\right\|_{L_{\#}^{2}}^{2}\right)^{1 / 2} .
\end{aligned}
$$


Let us now estimate $\sum_{j=1}^{N}\left\|\phi_{j, N_{c}}^{(1)}\right\|_{L_{\#}^{2}}^{2}$. Using (3.6)-(3.7) and noting that $\Pi_{N_{\mathrm{c}}}^{\perp}$ and $(1-\Delta)^{-1 / 2}$ commute, we get

$$
\begin{aligned}
\sum_{i=1}^{N}\left\|\phi_{i, N_{\mathrm{c}}}^{(1)}\right\|_{L_{\#}^{2}}^{2} & =\sum_{i=1}^{N}\left\|\left(-\frac{1}{2} \Delta-\lambda_{i, N_{\mathrm{c}}}\right)^{-1} \Pi_{N_{\mathrm{c}}}^{\perp} \mathcal{V}_{N_{\mathrm{c}}}^{\perp} \phi_{i, N_{\mathrm{c}}}\right\|_{L_{\#}^{2}}^{2} \\
& \leq \max _{i=1, \cdots, N}\left\|\left(-\frac{1}{2} \Delta-\lambda_{i, N_{\mathrm{c}}}\right)^{-1}(1-\Delta)^{1 / 2} \Pi_{N_{\mathrm{c}}}^{\perp}\right\|^{2} \sum_{i=1}^{N}\left\|(1-\Delta)^{-1 / 2} \mathcal{V}_{N_{\mathrm{c}}}^{\perp} \phi_{i, N_{\mathrm{c}}}\right\|_{L_{\#}^{2}}^{2} \\
& \leq\left(1+N_{\mathrm{c}}^{2}\right)^{-1} \max _{i=1, \cdots, N}\left\|\left(-\frac{1}{2} \Delta-\lambda_{i, N_{\mathrm{c}}}\right)^{-1}(1-\Delta)\right\|^{2} \sum_{i=1}^{N}\left\|(1-\Delta)^{-1 / 2} \mathcal{V}_{N_{\mathrm{c}}}^{\perp} \phi_{i, N_{\mathrm{c}}}\right\|_{L_{\#}^{2}}^{2} \\
& =\left(1+N_{\mathrm{c}}^{2}\right)^{-1} \max _{i=1, \cdots, N}\left\|\left(-\frac{1}{2} \Delta-\lambda_{i, N_{\mathrm{c}}}\right)^{-1}(1-\Delta)\right\|^{2}\left\|(1-\Delta)^{-1 / 2} \mathcal{V}_{N_{\mathrm{c}}}^{\perp} \gamma_{0, N_{\mathrm{c}}}\right\|_{\mathfrak{S}_{2}\left(L_{\#}^{2}\right.}^{2} .
\end{aligned}
$$

Therefore, since $\max _{i=1, \cdots, N}\left\|\left(-\frac{1}{2} \Delta-\lambda_{i, N_{\mathrm{c}}}\right)^{-1}(1-\Delta)\right\|^{2}$ is bounded uniformly in $N_{\mathrm{c}}$, we deduce from (4.11) that there exist $C \in \mathbb{R}_{+}$and $N_{\mathrm{c}}^{0} \in \mathbb{N}$, such that for all $N_{\mathrm{c}} \geq N_{\mathrm{c}}^{0}$,

$$
\sum_{i=1}^{N}\left\|\phi_{i, N_{\mathrm{c}}}^{(1)}\right\|_{L_{\#}^{2}}^{2} \leq C N_{\mathrm{c}}^{-2}\left\|(1-\Delta)^{1 / 2}\left(\gamma_{0, N_{\mathrm{c}}}-\gamma_{0}\right)\right\|_{\mathfrak{S}_{2}\left(L_{\#}^{2}\right.}^{2} .
$$

From (2.24), (2.27) and (4.21), we obtain that there exist $C \in \mathbb{R}_{+}$and $N_{\mathrm{c}}^{0} \in \mathbb{N}$, such that for all $N_{\mathrm{c}} \geq N_{\mathrm{c}}^{0}$,

$$
\left|\operatorname{Tr}\left(\gamma_{0}\left(\mathcal{H}-\epsilon_{\mathrm{F}}\right) \gamma_{N_{\mathrm{c}}}^{(1)}\right)\right| \leq C N_{\mathrm{c}}^{-2}\left\|(1-\Delta)^{1 / 2}\left(\gamma_{0, N_{\mathrm{c}}}-\gamma_{0}\right)\right\|_{\mathfrak{S}_{2}\left(L_{\#}^{2}\right)}^{2} .
$$

Putting together (4.18), (4.19), (4.20), (4.22), and (2.18), we obtain estimate (4.2).

\section{$5 \quad$ Numerical results}

We present in this section some results to illustrate the statements of Theorem 4.1 for several eigenvalue clusters and potentials with different regularities. First note that the quantities $\phi_{j, N_{\mathrm{c}}}^{(1)}$ defined in (3.6) are easily computable. Indeed, the operator $\left(-\frac{1}{2} \Delta-\lambda_{j, N_{c}}\right)$ is diagonal in plane-wave bases, hence very easy to invert. Moreover, only two FFT's are needed to compute the residual or $\nu_{N_{\mathrm{c}}}^{\perp} \phi_{j, N_{\mathrm{c}}}$ on a larger grid, via a product in the physical space. Since we demonstrated the improvement brought by the method as well as its low computational cost on the nonlinear problem of Kohn-Sham equations in [7], we focus in this article on the convergence rate improvement that we illustrate on examples for which we explicitely control the regularity.

In all what follows, we consider a domain $\Omega=(0,10)^{3}$ in atomic units (a.u.). The computed solutions are compared to a reference solution, computed in a very large basis with a kinetic energy cutoff $E_{\text {ref }}=800$ a.u., which corresponds to a discretization parameter $N_{\text {ref }} \simeq 58.5$, and 382,323 Fourier basis functions. In each case, we denote the reference energy by $\varepsilon_{0}$ and the reference density matrix by $\gamma_{0}$.

The coarse solutions are computed in a basis with cutoff $E_{c}$ and corresponding $N_{c}$, and have energy $\varepsilon_{0, N_{\mathrm{c}}}$ and density matrix $\gamma_{0, N_{\mathrm{c}}}$. In order to avoid errors coming from the size of the finite basis used for the computation of the corrections, we compute the post-processed solutions in the same basis as the reference

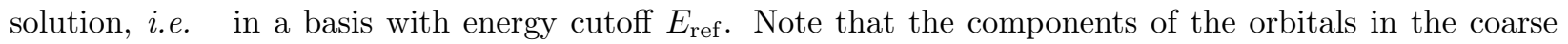
basis are not modified by the post-processing. One only needs to compute the coefficients corresponding to Fourier modes with kinetic energy larger than $E_{c}$.

The implementation is based on KSSOLV [23], a Matlab library for solving Kohn-Sham equations, which we use here to solve the linear eigenvalue problem (3.3).

The tested potentials denoted by $\mathcal{V}_{s}$ are defined by their Fourier coefficients as

$$
\widehat{\mathcal{V}}_{s, \mathbf{0}}=0, \quad \text { and } \quad \forall \mathbf{k} \in \mathcal{R}^{*} \backslash\{\mathbf{0}\}, \quad \widehat{\mathcal{V}}_{s, \mathbf{k}}=-\frac{c_{s}}{|\mathbf{k}|^{2 s}},
$$


where $s \in[1,2]$ is a regularity parameter, and $c_{s}$ is a multiplicative constant. For $s>3 / 2$, the potential $\mathcal{V}_{s}$ is smooth enough to verify Assumption 2.2, hence we expect to observe the improvement in the convergence rate given in Theorem 4.1. For $s \leq 3 / 2$, the potential does not verify Assumption 2.2, but we can nevertheless compute post-processed eigenfunctions and eigenvalues. As we will see, it actually still yields an improvement on the energy and the density matrix. Note that for $s=1$, this potential has the same regularity as the Coulomb potential.

The lowest eigenvalue of the Hamiltonian $-\frac{1}{2} \Delta+\nu$ is simple. For all the tested potentials, there are gaps between the $5^{\text {th }}$ and $6^{\text {th }}$ eigenvalues and the $10^{\text {th }}$ and $11^{\text {th }}$ eigenvalues. Therefore, in the following, we present the results of the post-processing method for clusters including one, five, and ten eigenvalues. This guarantees that the gap Assumption 2.1 is satisfied. Let us remind that we consider the cluster of lowest eigenvalues of the Hamiltonian.

In Subsection 5.1, we show how the post-processing procedure decreases both the energy error $\mathcal{E}_{0, N_{\mathrm{c}}}-\mathcal{E}_{0}$ and the energy norm $\left\|(1-\Delta)^{1 / 2}\left(\gamma_{0, N_{\mathrm{c}}}-\gamma_{0}\right)\right\|_{\mathfrak{S}_{2}\left(L_{\#}^{2}\right)}$ of the density matrix error in the case of a potential with regularity parameter $s=2$ and a cluster of five eigenvalues. In Subsection 5.2, we study the convergence rate improvement of both the energy and the density matrix for different clusters of eigenvalues, still in the case of a potential with regularity parameter $s=2$. Finally, we study in Subsection 5.3 the influence of the potential regularity $s$ on the convergence rate improvement for the energy and the density matrix, in the case of the cluster composed of the lowest five eigenvalues.

\subsection{Convergence of the density matrix and the energy}

We consider the potential $\nu_{2}$ with Fourier coefficients

$$
\widehat{\mathcal{V}}_{2, \mathbf{0}}=0, \quad \text { and } \quad \forall \mathbf{k} \in \mathcal{R}^{*} \backslash\{\mathbf{0}\}, \quad \widehat{\mathcal{V}}_{2, \mathbf{k}}=-\frac{0.01}{|\mathbf{k}|^{4}} .
$$

For all energy cutoffs $E_{c}$ between 10 and 200 a.u. by steps of 10 , we compute the lowest five eigenvalues and eigenvectors of the discrete Hamiltonian. We build the discrete density matrix $\gamma_{0, N_{\mathrm{c}}}$ as in (2.21), and compute the discrete energy $\mathcal{E}_{0, N_{\mathrm{c}}}(2.22)$. Then, we apply the post-processing as described in Section 3 and we compute the post-processed density matrix $\widetilde{\gamma_{0, N_{\mathrm{c}}}}$ as well as the perturbed energy $\widetilde{\varepsilon_{0, N_{\mathrm{c}}}}$. For the potential, we choose such a small multiplicative constant $\left(c_{s}=0.01\right)$ to better observe the asymptotic regime numerically within the range of tested cutoffs $E_{\mathrm{c}}$.

As we can see on the top part of Figure 1, the energy error between the post-processed energy and the reference energy $\widetilde{\varepsilon_{0, N_{\mathrm{c}}}}-\mathcal{E}_{0}$ is 5 to 50 times smaller than the energy error between the coarse energy and the reference energy $\varepsilon_{0, N_{\mathrm{c}}}-\mathcal{E}_{0}$. More precisely, the energy error is reduced by a factor of about 5 for small values of $N_{\mathrm{c}}$ and up to 50 for large values of $N_{\mathrm{c}}$.

We observe a similar behavior for the density matrix error on the bottom part of Figure 1. Indeed, the Hilbert-Schmidt norm of the difference between the reference and the coarse density matrices $\|(1-$ $\Delta)^{1 / 2}\left(\gamma_{0}-\gamma_{0, N_{\mathrm{c}}}\right) \|_{\mathfrak{S}_{2}\left(L_{\#}^{2}\right)}$ is 5 to 50 times larger than the error between the reference and the post-processed density matrix $\left\|(1-\Delta)^{1 / 2}\left(\gamma_{0}-\widetilde{\gamma_{0, N_{\mathrm{c}}}}\right)\right\|_{\mathfrak{S}_{2}\left(L_{\#}^{2}\right)}$.

\subsection{Comparison between different eigenvalue clusters}

We now consider three different eigenvalue clusters composed of one, five and ten eigenvalues, still with the same potential $\mathcal{V}_{2}$. The three corresponding gaps are respectively equal to $8.84 \cdot 10^{-1}, 1.80 \cdot 10^{-1}$ and $3.42 \cdot 10^{-1}$.

For these three clusters, we compute a reference solution, and then we compute discrete solutions within cutoffs $E_{c}$ varying between 10 and 200 a.u. On the top of Figure 2, we plot the ratio between the energy error with post-processing and without post-processing $\frac{\widehat{\varepsilon_{0, N_{\mathrm{N}}}-\varepsilon_{0}}}{\frac{\varepsilon_{0}, \varepsilon_{\mathrm{c}}}{-}-\varepsilon_{0}}$ for the three different cases. According to Theorem 4.1, this ratio should at least decrease as $N_{\mathrm{c}}^{-2}$ in the asymptotic regime of large $N_{\mathrm{c}}$ 's. This is approximately satisfied with the decay $N_{\mathrm{c}}^{-1.74}$ observed in the numerical simulations. The difference 

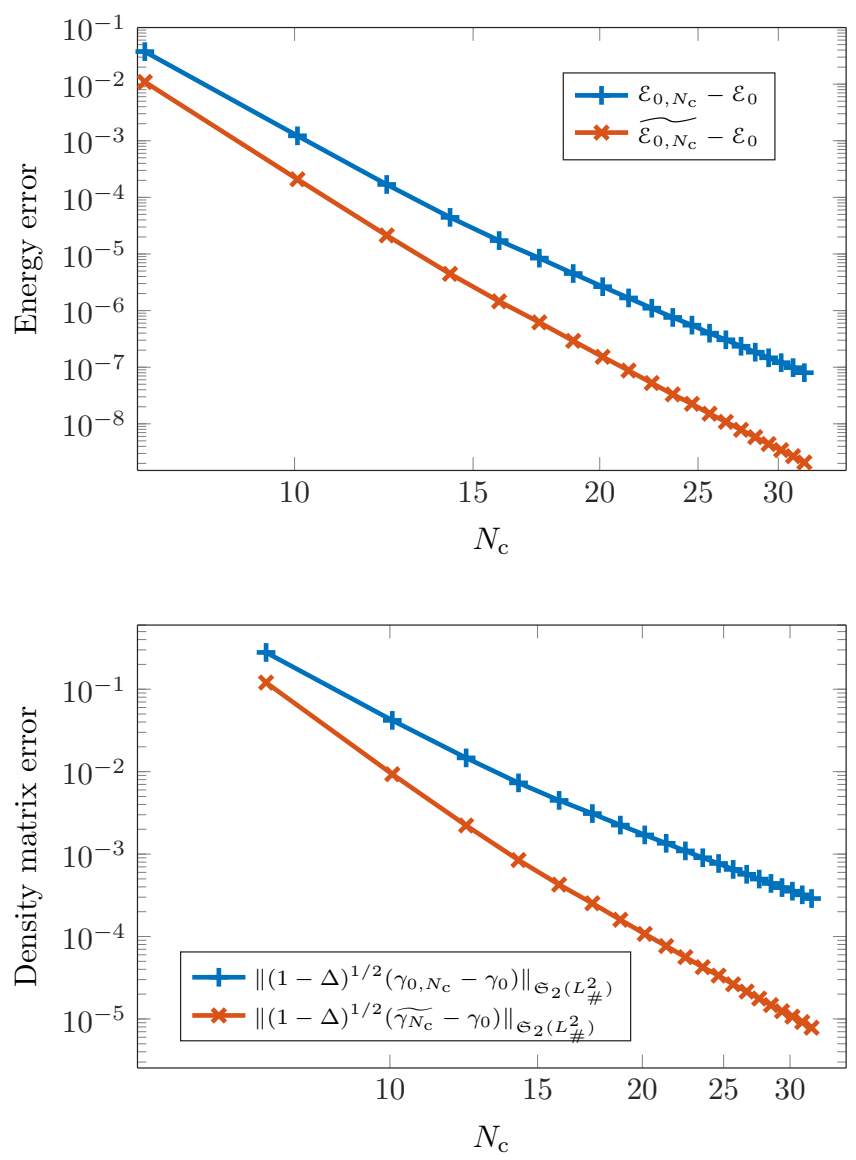

Figure 1: Top: plot of the energy errors $\widetilde{\mathcal{E}_{0, N_{\mathrm{c}}}}-\varepsilon_{0}$ and $\mathcal{E}_{0, N_{\mathrm{c}}}-\varepsilon_{0}$ for energy cutoffs $E_{c}$ between 10 and 200 a.u. Bottom: plot of the density matrix errors $\left\|(1-\Delta)^{1 / 2}\left(\gamma_{0}-\widetilde{\gamma_{0, N_{c}}}\right)\right\|_{\mathfrak{S}_{2}\left(L_{\#}^{2}\right)}$ and $\left\|(1-\Delta)^{1 / 2}\left(\gamma_{0}-\gamma_{0, N_{\mathrm{c}}}\right)\right\|_{\mathfrak{S}_{2}\left(L_{\#}^{2}\right)}$ for energy cutoffs between 10 and 200 a.u. This corresponds to values of $N_{c}$ between 7 and 31 .

between the expected and observed rate might come from a pre-asymptotic effect. Likewise, the ratio

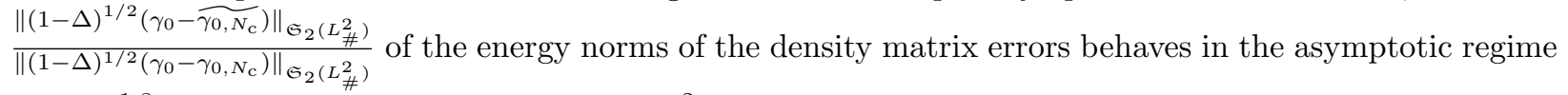
like $N_{\mathrm{c}}^{-1.8}$ for one and five eigenvalues, and $N_{\mathrm{c}}^{-2}$ for the cluster with ten eigenvalues, as shown on the bottom of Figure 2.

In Figure 2, the preconstant in the improvement factor does not seem to depend on the number of computed eigenvectors $N$, while the theoretical constants in Theorems 4.1 can depend on $N$, even superlinearly. This suggests that the sharpness of the bounds with respect to $N$ could be improved. This is left for future work.

\subsection{Comparison of different regularities}

Lastly, we perform the post-processing in the case of the cluster containing the lowest five eigenvalues with four potentials having different regularity coefficients. More precisely, we consider the potentials $\mathcal{V}_{1}, \mathcal{V}_{1.25}, \mathcal{V}_{1.5}$, and $\mathcal{V}_{2}$, again taking $c_{s}=0.01$. In theory, the potential $\mathcal{V}_{2}$ satisfies Assumption 2.2 , the 

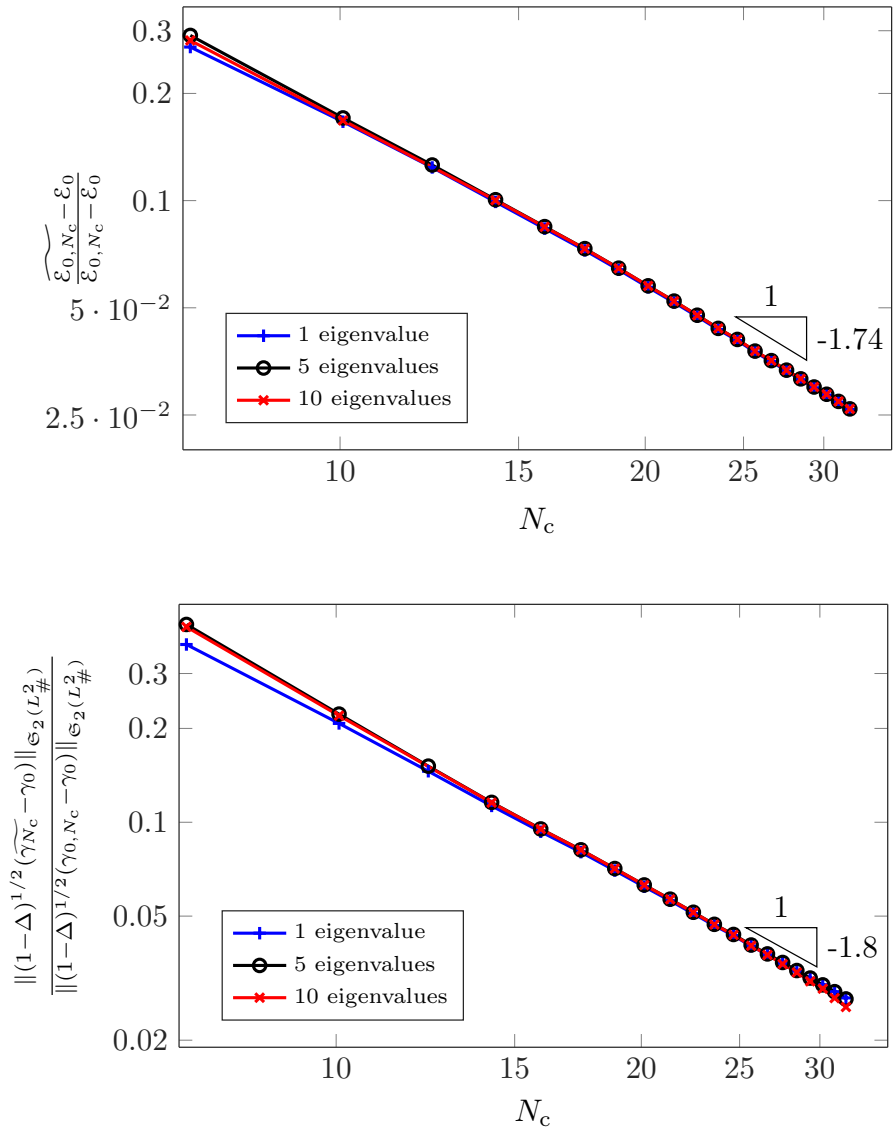

Figure 2: Plots of the energy error ratio (top) and the density matrix error ratio (bottom) for three different clusters of eigenvalues (1, 5 and 10 eigenvalues) with a potential with regularity coefficient $s=2$. The convergence rates are computed with the largest 10 values of $N_{\mathrm{c}}$ respectively yielding to $N_{\mathrm{c}}^{-1.73}, N_{\mathrm{c}}^{-1.74}$, $N_{\mathrm{c}}^{-1.74}$ (top), and $N_{\mathrm{c}}^{-1.79}, N_{\mathrm{c}}^{-1.84}, N_{\mathrm{c}}^{-2.01}$ (bottom). 

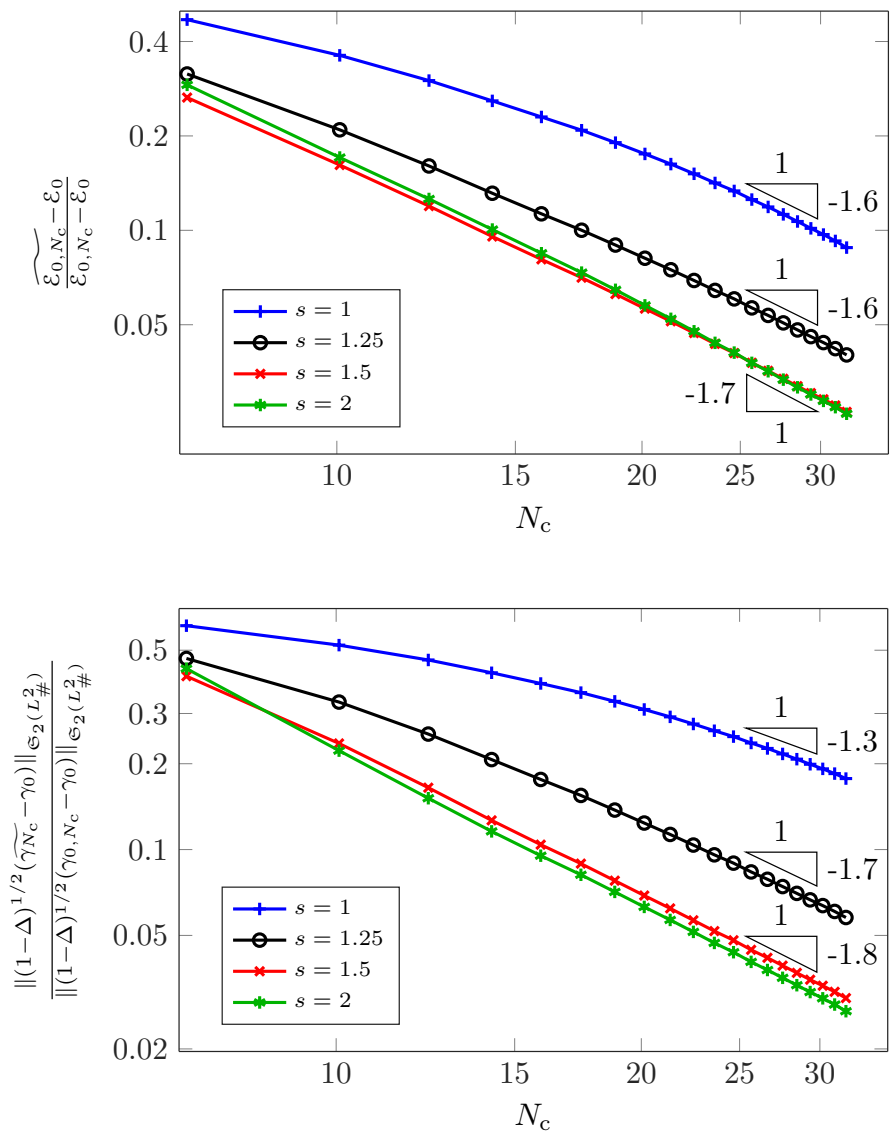

Figure 3: Plots of the energy error improvement (top) and the density matrix error improvement (bottom) for four different regularities for the potential: $s=1,1.25,1.5,2$, cluster of five eigenvalues. The convergence rates are computed with the largest 10 values of $N_{\mathrm{c}}$ respectively yielding to $N_{\mathrm{c}}^{-1.59}, N_{\mathrm{c}}^{-1.58}, N_{\mathrm{c}}^{-1.67}, N_{\mathrm{c}}^{-1.74}$ (top), and $N_{\mathrm{c}}^{-1.29}, N_{\mathrm{c}}^{-1.69}, N_{\mathrm{c}}^{-1.81}, N_{\mathrm{c}}^{-1.84}$ (bottom).

potential $\mathcal{V}_{1.5}$ is just at the limit, and $\mathcal{V}_{1.25}, \mathcal{V}_{1}$ do not satisfy the assumption. It is nevertheless possible to compute the post-processed energy and density matrix for each of these potentials. Numerically, we observe an improved convergence rate both for the energy error ratio (on the top of Figure 3) and for the density matrix error ratio (on the bottom of Figure 3) for all potentials, which slowly and monotonically decreases as the regularity of the potential decreases. Hence, this post-processing method seems to yield an improvement also in a larger setting than what is covered by the proof of this article. Also, note that for the potentials $\mathcal{V}_{1.25}$ and $\mathcal{V}_{1}$ with low regularity, the tested $E_{\mathrm{c}}$ are far from convergence, so the asymptotic regime might not have been reached. 


\section{Appendix: proof of Lemma 2.1}

We start by proving (2.24). Denoting by $M_{N_{\mathrm{c}}}$ the $N \times N$ overlap matrix with entries $\left(M_{N_{\mathrm{c}}}\right)_{i, j}=\left\langle\phi_{i, N_{\mathrm{c}}}^{0} \mid \phi_{j}^{0}\right\rangle$, we have

$$
\left\|\gamma_{0, N_{\mathrm{c}}}-\gamma_{0}\right\|_{\mathfrak{S}_{2}\left(L_{\#}^{2}\right)}^{2}=2\left(N-\sum_{i, j=1}^{N}\left|\left\langle\phi_{j, N_{\mathrm{c}}}^{0} \mid \phi_{i}^{0}\right\rangle\right|^{2}\right)=2\left(N-\operatorname{Tr}\left(M_{N_{\mathrm{c}}} M_{N_{\mathrm{c}}}^{T}\right)\right)
$$

and

$$
\left\|\Phi_{N_{\mathrm{c}}}^{0}-\Phi^{0}\right\|_{L_{\#}^{2}}^{2}=2\left(N-\sum_{i=1}^{N}\left\langle\phi_{i, N_{\mathrm{c}}}^{0} \mid \phi_{i}^{0}\right\rangle\right)=2\left(N-\operatorname{Tr}\left(M_{N_{\mathrm{c}}}\right)\right) .
$$

We therefore have to show that

$$
2 N-2 \operatorname{Tr}\left(M_{N_{\mathrm{c}}}\right) \leq 2 N-2 \operatorname{Tr}\left(M_{N_{\mathrm{c}}} M_{N_{\mathrm{c}}}^{T}\right) \leq 2\left(2 N-2 \operatorname{Tr}\left(M_{N_{\mathrm{c}}}\right)\right) .
$$

Since $\Phi_{N_{\mathrm{c}}}^{0}$ belongs to $\mathcal{M}^{\Phi^{0}}$, we have from [3, Lemma 4.3]

$$
M_{N_{\mathrm{c}}}=M_{N_{\mathrm{c}}}^{T}=\left(M_{N_{\mathrm{c}}} M_{N_{\mathrm{c}}}^{T}\right)^{1 / 2} \text { and } \quad 0 \leq M_{N_{\mathrm{c}}} \leq 1 .
$$

Hence,

$$
\operatorname{Tr}\left(M_{N_{\mathrm{c}}} M_{N_{\mathrm{c}}}^{T}\right)=\operatorname{Tr}\left(M_{N_{\mathrm{c}}}^{2}\right) \leq \operatorname{Tr}\left(M_{N_{\mathrm{c}}}\right)
$$

from which we deduce the left inequality in (2.24). The right inequality in (2.24) holds since

$$
2 N-4 \operatorname{Tr}\left(M_{N_{\mathrm{c}}}\right)+2 \operatorname{Tr}\left(M_{N_{\mathrm{c}}} M_{N_{\mathrm{c}}}^{T}\right)=2 N-4 \operatorname{Tr}\left(M_{N_{\mathrm{c}}}\right)+2 \operatorname{Tr}\left(M_{N_{\mathrm{c}}}^{2}\right)=2 \operatorname{Tr}\left(\left(M_{N_{\mathrm{c}}}-I_{N}\right)^{2}\right) \geq 0 .
$$

Let us now show (2.25). From [3, Theorem 4.2], there exist two constants $0<c \leq C<\infty$ and $N_{\mathrm{c}}^{0} \in \mathbb{N}$ such that for all $N_{\mathrm{c}} \geq N_{\mathrm{c}}^{0}$,

$$
c\left\|(1-\Delta)^{1 / 2}\left(\Phi_{N_{\mathrm{c}}}^{0}-\Phi^{0}\right)\right\|_{L_{\#}^{2}}^{2} \leq \mathcal{E}_{0, N_{\mathrm{c}}}-\mathcal{E}_{0} \leq C\left\|(1-\Delta)^{1 / 2}\left(\Phi_{N_{\mathrm{c}}}^{0}-\Phi^{0}\right)\right\|_{L_{\#}^{2}}^{2} .
$$

Hence, using (2.18) finishes the proof.

\section{Acknowledgements}

This work was supported by the ANR project MANIF Mathematical and numerical issues in first-principle molecular simulation. Part of this work has been supported from French state funds managed by the CalSimLab LABEX and the ANR within the Investissements d'Avenir program (reference ANR-11-LABX-0037-01). MV has also received funding from the European Research Council (ERC) under the European Union's Horizon 2020 research and innovation program (Grant Agreement No. 647134 GATIPOR). BS acknowledges the funding from the German Academic Exchange Service (DAAD) from funds of the Bundesministeriums für Bildung und Forschung (BMBF) for the project Aa-Par-T (Project-ID 57317909). YM and EC acknowledge funding from PICS-CNRS, PHC PROCOPE 2017 (Project No. 37855ZK). This work has received funding from the European Research Council (ERC) under the European Union's Horizon 2020 research and innovation programme (grant agreement No 810367). Part of this work was supported by the French "Investissements d'Avenir" program, project ISITE-BFC (contract ANR-15-IDEX-0003). 


\section{References}

[1] M. Born and R. Oppenheimer, Zur Quantentheorie der Molekeln, Annalen der Physik, 389 (1927), pp. $457-484$.

[2] E. Cancès, R. Chakir, L. He, and Y. Maday, Two-grid methods for a class of nonlinear elliptic eigenvalue problems, IMA J. Numer. Anal., 38 (2018), pp. 605-645.

[3] E. CANCÈs, R. ChakiR, AND Y. MAdAy, Numerical analysis of the planewave discretization of some orbital-free and Kohn-Sham models, ESAIM: Mathematical Modelling and Numerical Analysis, 46 (2012), pp. 341-388.

[4] E. Cancès, M. Defranceschi, W. Kutzelnigg, C. Le Bris, and Y. Maday, Computational quantum chemistry: A primer, in Handbook of Numerical Analysis, vol. 10, Elsevier, Jan. 2003, pp. 3270 .

[5] E. Cancès, A. Deleurence, And M. Lewin, A new approach to the modeling of local defects in crystals: The reduced Hartree-Fock case, Communications in Mathematical Physics, 281 (2008), pp. 129177 .

[6] E. Cancès, G. Dusson, Y. Maday, B. Stamm, and M. Vohralík, A perturbation-method-based a posteriori estimator for the planewave discretization of nonlinear Schrödinger equations, Comptes Rendus Mathematique, 352 (2014), pp. 941-946.

[7] _ - A perturbation-method-based post-processing for the planewave discretization of Kohn-Sham models, Journal of Computational Physics, 307 (2016), pp. 446-459.

[8] F. Chatelin, Spectral Approximation of Linear Operators, Computer science and applied mathematics, Academic Press, 1983.

[9] R. M. Dreizler and E. K. U. Gross, Density Functional Theory, Springer Berlin Heidelberg, Berlin, Heidelberg, 1990.

[10] G. Dusson, Post-processing of the planewave approximation of Schrödinger equations. Part II: KohnSham models, (in preparation). 2019.

[11] G. Dusson And Y. Maday, An overview of a posteriori estimation and post-processing methods for nonlinear eigenvalue problems, (in preparation). 2020.

[12] T. Helgaker, P. Jørgensen, and J. Olsen, Molecular Electronic Structure Theory, John Wiley \& Sons, LTD, Chichester, 2000.

[13] P. Henning, A. Målqvist, and D. Peterseim, Two-level discretization techniques for ground state computations of Bose-Einstein condensates, SIAM J. Numer. Anal., 52 (2014), pp. 1525-1550.

[14] M. Hoffmann-Ostenhof and T. Hoffmann-Ostenhof, "Schrödinger inequalities" and asymptotic behavior of the electron density of atoms and molecules, Phys. Rev. A, 16 (1977), pp. 1782-1785.

[15] T. Kato, Perturbation Theory for Linear Operators, Classics in Mathematics, Springer Berlin Heidelberg, Berlin, Heidelberg, 1976.

[16] W. Kohn and L. J. Sham, Self-consistent equations including exchange and correlation effects, Physical Review, 140 (1965), pp. A1133-A1138.

[17] W. A. Lester, Recent Advances in Quantum Monte Carlo Methods, vol. 2 of Recent Advances in Computational Chemistry, World Scientific, 1997. 
[18] W. A. Lester, S. M. Rothstein, And S. Tanaka, Recent Advances in Quantum Monte Carlo Methods - Part II, vol. 2 of Recent Advances in Computational Chemistry, World Scientific, 2002.

[19] Y. Maday And G. TuRinici, Error bars and quadratically convergent methods for the numerical simulation of the Hartree-Fock equations, Numerische Mathematik, 94 (2003), pp. 739-770.

[20] M. Reed And B. Simon, Methods of Modern Mathematical Physics, Vol. 1: Functional Analysis, Academic Press, 1972.

[21] M. Reed And B. Simon, Methods of modern mathematical physics, Vol. 2: Fourier analysis, selfadjointness, Academic Press, 1975.

[22] J. Xu And A. Zhou, A two-grid discretization scheme for eigenvalue problems, Mathematics of Computation, 70 (1999), pp. 17-26.

[23] C. Yang, J. C. Meza, B. Lee, and L.-W. WAng, KSSOLV-a MATLAB toolbox for solving the Kohn-Sham equations, ACM Transactions on Mathematical Software, 36 (2009), pp. 1-35. 